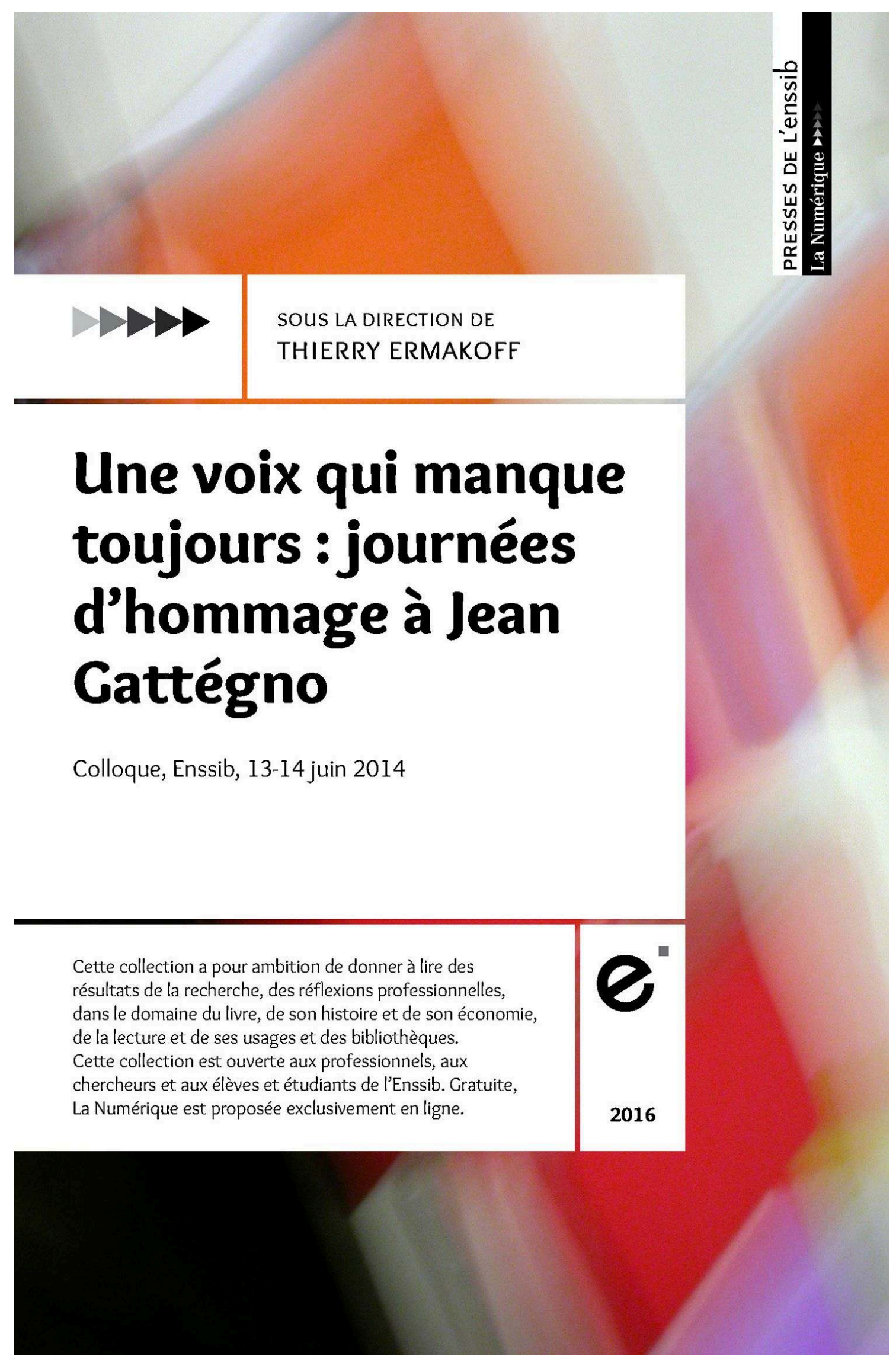




\section{Une voix qui manque toujours : journées d'hommage à Jean Gattégno}

\section{Thierry Ermakoff (dir.)}

DOI : 10.4000/books.pressesenssib. 1775

Éditeur : Presses de l'enssib

Lieu d'édition : Villeurbanne

Année d'édition : 2016

Date de mise en ligne : 4 avril 2017

Collection : La Numérique

ISBN électronique : 9782375460528

\section{Sbooks}

http://books.openedition.org

\section{Référence électronique}

ERMAKOFF, Thierry (dir.). Une voix qui manque toujours : journées d'hommage à Jean Gattégno. Nouvelle édition [en ligne]. Villeurbanne : Presses de l'enssib, 2016 (généré le 01 février 2021). Disponible sur Internet : <http://books.openedition.org/pressesenssib/1775>. ISBN : 9782375460528 . DOI : https:// doi.org/10.4000/books.pressesenssib.1775.

(c) Presses de l'enssib, 2016

Conditions d'utilisation

http://www.openedition.org/6540 


\section{Une voix qui manque} toujours : journées d'hommage

\section{à Jean Gattégno}

Colloque à l'Enssib organisé les 13 et 14 juin 2014

Mots clés : bibliothèques publiques, CNL (centre national du livre), décentralisation, économie du livre, éditeurs, Jean Gattégno, homosexualités, Ombres Blanches, politiques publiques

Cette collection a pour ambition de donner à lire des résultats de la recherche, des réflexions professionnelles, dans le domaine du livre, de son histoire et de son économie, de la lecture et de ses usages et des bibliothèques. Cette collection est ouverte aux professionnels, aux chercheurs et aux élèves et étudiants de l'Enssib.

Gratuite, La Numérique est proposée exclusivement en ligne. 


\section{L'ouvrage}

Jean Gattégno, spécialiste de littérature anglaise, haut fonctionnaire, ancien directeur du livre et de la lecture de 1981 à 1989, nous a quittés le 31 mai 1994. Les 13 et 14 juin 2014, deux journées d'hommage lui ont été rendues à l'Enssib.

\section{Les auteurs}

\begin{tabular}{|c|c|}
\hline & $\begin{array}{l}\text { Directeur d'études à l'École } \\
\text { des hautes études en sciences } \\
\text { sociales }\end{array}$ \\
\hline $\begin{array}{l}\text { Véronique } \\
\text { Chatenay-Dolto }\end{array}$ & $\begin{array}{l}\text { Directrice régionale des affaires } \\
\text { culturelles d'Ile-de-France }\end{array}$ \\
\hline Nicolas Galaud & $\begin{array}{l}\text { Directeur de la bibliothèque } \\
\text { municipale de Brest }\end{array}$ \\
\hline Gérard Grunberg & $\begin{array}{l}\text { Professeur associé à l'Université } \\
\text { Senghor }\end{array}$ \\
\hline Denis Llavori & $\begin{array}{l}\text { Directeur de l'action culturelle } \\
\text { au Conseil départemental du } \\
\text { Cantal, Aurillac }\end{array}$ \\
\hline François Suard & $\begin{array}{l}\text { Professeur émérite à Paris } \\
\text { Ouest }\end{array}$ \\
\hline Valérie Tesnière & $\begin{array}{l}\text { Directrice de la Bibliothèque } \\
\text { de documentation internatio- } \\
\text { nale contemporaine (BDIC), } \\
\text { Nanterre }\end{array}$ \\
\hline Christian Thorel & $\begin{array}{l}\text { Responsable de la librairie } \\
\text { Ombres blanches, Toulouse }\end{array}$ \\
\hline Benoît Tuleu & $\begin{array}{l}\text { Directeur du département } \\
\text { du Dépôt légal, Bibliothèque } \\
\text { nationale de France, Paris }\end{array}$ \\
\hline
\end{tabular}




\section{Notice}

Une voix qui manque toujours : journées d'hommage à Jean Gattégno / sous la direction de Thierry Ermakoff.

- Villeurbanne : Presses de l'enssib,

cop. 2016. - 1 vol. (81 p.).

- (La Numérique ; ISSN 2492-9735)

ISBN pdf. 978-2-37546-002-3. Gratuit

ISBN epub. 978-2-37546-003-0. Gratuit.

Dewey : 920.71 ou 027.92

\section{Rameau :}

Gattégno, Jean (1935-1994) - Mélanges et hommages Livres - Histoire

Bibliothèques -- Décentralisation

Bibliothèques - Histoire

Culture - Aspect économique

Librairie - France - 1990

Politique culturelle

(9)(1)(ङ 2016. 


\section{Sommaire}

Présentation de l'ouvrage $\quad 2$

Les auteurs $\quad 2$

Notice bibliographique $\quad 3$

1. Jean Gattégno, les institutions et le service public (1981-1994), par Marc Olivier Baruch 6

2. Bibliothèques et développement de la culture, par Gérald Grunberg

3. L'engagement scientifique de Jean Gattégno dans les bibliothèques, par Valérie Tesnière $\quad 22$

1. Lecture publique? 22

2. Usages de la lecture 25

3. Encyclopédisme, pluridisciplinarité, interdisciplinarité 28

4. Vingt ans après, par Nicolas Galaud 34

5. Peut-on, aujourd'hui encore, être fidèle à la pensée de Jean Gattégno ?, par Denis Llavori 45

1. Introduction 45

2. Développements 48

3. Conclusion 53

6. Trente-trois ans après sa publication, relire « Du pécheur au militant ", par Benoît Tuleu 55

7. Jean Gattégno et la foi chrétienne, dialogue entre Véronique Chatenay-Dolto et François Suard

1. L'origine d'une vocation, le sens d'un engagement

2. L'héritage de Jean Gattégno, le chemin d'un homme parmi les hommes de bonne volonté

8. Le livre n'est pas un produit comme les autres, par Christian Thorel 


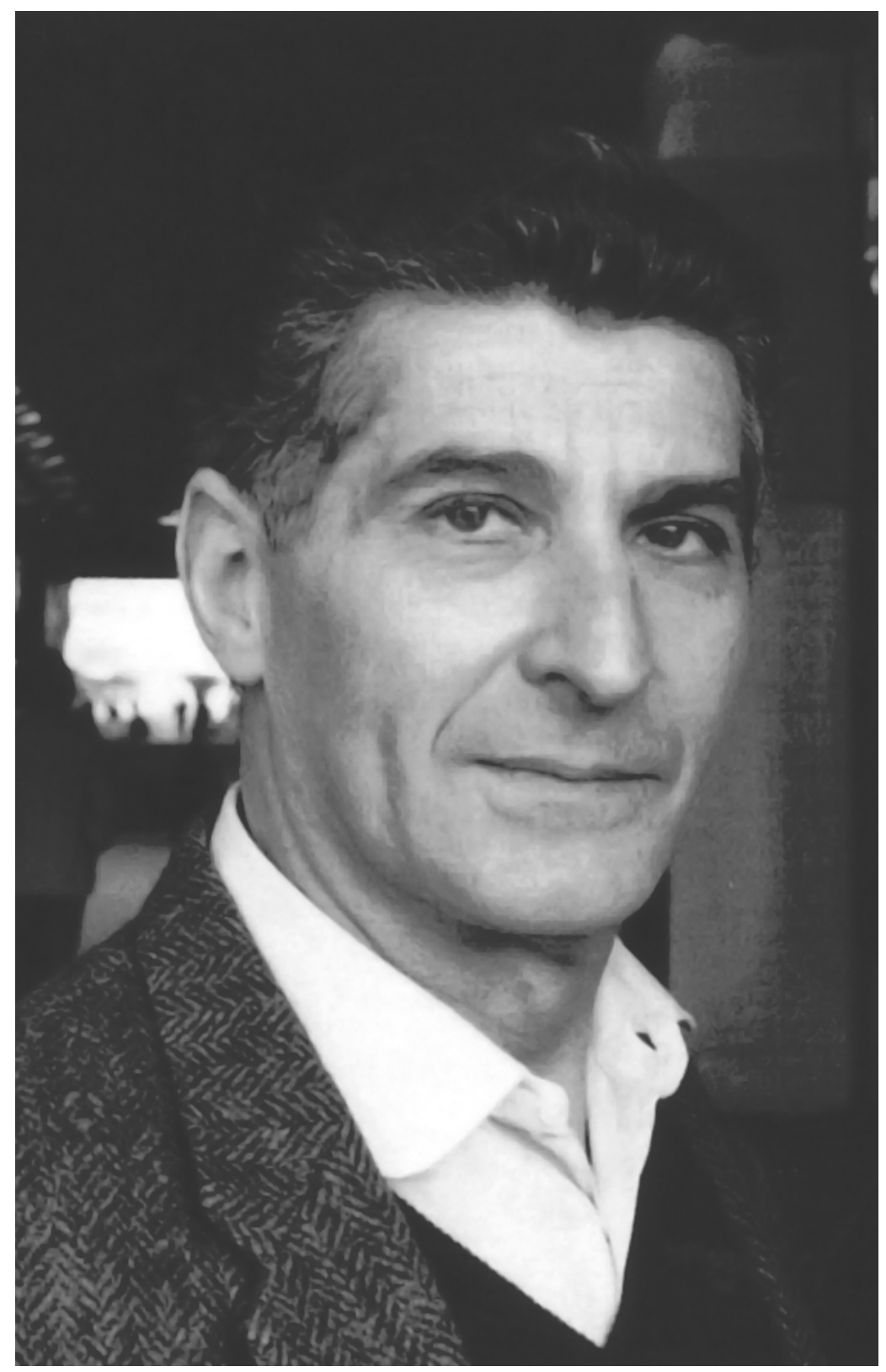

Source : (c) Pierre-Olivier Deschamps / VU’ 


\section{Jean Gattégno, les institutions et le service public (1981-1994)}

par Marc Olivier Baruch

Je remercie avec chaleur l'École nationale supérieure des sciences de l'information et des bibliothèques (Enssib), et avec eux tous ceux qui ont œuvré à la réussite de ces journées. Comme je remercie les organismes, publics ou privés, qui nous ont aidés : la région Rhône-Alpes, la Société française des intérêts des auteurs de l'écrit (Sofia), le Centre national du livre (CNL), le comité d'histoire du ministère de la Culture, l'Institut national de l'audiovisuel (INA) et sa délégation Centre-Est, Livres Hebdo.

Qu'ont-ils fait, les uns ou les autres ? Ils n'ont pas demandé, ils n'ont pas exigé, ils n'ont pas compliqué ; ils ont proposé, ils ont aidé, ils ont facilité. Parce que, me semble-t-il, ils ont estimé qu’à un tel hommage l'institution qu'ils représentaient aujourd'hui se devait d'être associée. Soit qu'ils aient connu l'homme Jean Gattégno - «l'homme avec qualités » -, soit qu'ils aient compris que, « vingt ans après », l'œuvre accomplie alors continue de produire des effets.

En ouverture, l'exercice qui m'a été demandé est difficile : de traiter du rapport de Jean Gattégno aux institutions et au service public. Un peu parce que, comme le rappellerait tout professeur de droit public, ni la notion d'« institution ", ni - encore moins - celle de « service public » n'est parfaitement définie. Mais surtout parce qu'il est bien des épisodes de sa vie que je ne connais pas alors même qu'ils se sont situés dans des cadres institutionnels marquants.

D'autres évoqueront qu'il s'agisse - par ordre chronologique - de la rencontre avec l'Église catholique à l'École normale supérieure 
(deux institutions pas secondaires dans notre pays), de la guerre d'Algérie, de l'éducation populaire, de la coopération universitaire avec la Tunisie et par contrecoup de la dictature Bourguiba, de l'Université (pas n'importe laquelle : le Centre universitaire expérimental de Vincennes) et donc du gauchisme, du syndicalisme universitaire et corrélativement de la rencontre, du choc plutôt, avec l'appareil d'État giscardien, ici tenu par la terrible Alice Saunier-Seïté, qui fut pendant plus de cinq ans ministre des Universités.

Étant si ignorant, j'ai subrepticement glissé une parenthèse dans le titre de ce propos, qui devient Jean Gattégno, les institutions et le service public (1981-1994). Mais comme je suis historien, et que je sais qu'il n'y a jamais de début à quelque histoire que ce soit, il me faut évoquer d'abord une forme d'héritage. Tous ces moments que je viens de citer inscrivent le parcours et la personne de Jean Gattégno dans un monde institué et régulé, mais aussi dans un monde daté.

Daté non seulement par les années 1960 et 1970, celles de l'œuvre professionnelle et de l'engagement syndical, qui sont aussi celles de l'explosion académique et éditoriale des sciences sociales - notamment de la psychanalyse -, mais aussi par deux temps historiques : d'abord le XIX ${ }^{\text {e }}$ siècle, qui fut son domaine de recherche - siècle de la ville, de l'industrie et de la misère d'un côté, siècle du socialisme et de l'utopie de l'autre.

Et aussi, en résonance avec sa propre histoire, les tragiques années 1940 et la figure du général de Gaulle. Il m’indiqua un jour avoir toujours voté de Gaulle, qui restait l'homme du 18 juin. Pour de Gaulle en tant que personne j'imagine, donc votant sans doute encore "oui » au référendum d'avril 1969, mais je vois mal Jean Gattégno apportant sa voix à l'Union des démocrates pour la République (UDR) en juin 1968... Je ne sais pas. Après sa mort, alors que l'essentiel des livres de sa bibliothèque rejoignit 
Pont-en-Royans où fut inaugurée l'année suivante une médiathèque Jean Gattégno, je pris dans les rayonnages de la rue de Lyon la Lettre aux Anglais, de Bernanos - auteur que je n'avais jamais lu alors et que je n'ai jamais cessé de lire depuis. J'y vois, naïvement ou pire, un message, voire un commandement : ne pas se résigner !

Mais je m'égare : revenons à lui, au moyen d'une métaphore qui fait sens compte tenu de son ascendance. Sa palette, Jean Gattégno la composait à partir de trois couleurs primaires qui avaient pour noms : discipline, fraternité et responsabilité.

La discipline est celle qu'il s'imposait pour rendre compatible son métier de directeur d'administration centrale et président d'établissement public avec la poursuite d'une activité de chercheur et de traducteur. Sans doute écrivait-il facilement - sûrement pas aussi facilement qu'il parlait, car il dut être un grand professeur. Pour autant, quels que soient vos dons, le métier de chercheur exige de vous, en dose raisonnable, de la curiosité intellectuelle et de la vivacité d'esprit et, en dose moins raisonnable, du travail, du travail et du travail. Nous nous souvenons tous du « Il est 11 heures » qui indiquait, si l'on dînait chez lui, qu'il convenait de plier bagages et, s'il était invité, qu'il quittait les lieux. Car lui se lèverait tôt le lendemain pour traduire du Firbank ou du Wilde, rédiger une préface ou une notice pour la Pléiade.

J'appelle fraternité sa relation si facile, si engageante, si ouverte à l'autre. Bien sûr il aimait séduire, mais sans courtisanerie et surtout sans vanité. Bien sûr il pouvait avoir la dent dure, mais sans méchanceté et surtout sans dédain. Pas besoin de recourir à la psychanalyse plus ou moins sauvage pour imaginer que le petit garçon de 5 ans - en juin 1940 ! -, privé de mère et bientôt de famille, ait, sa vie durant, recherché puis construit des milieux fraternels : le confort d'une promotion de normaliens, l'enseignement non mandarinal de Vincennes, plus tard « l'aisance dans les 
contacts et le sens de la relation avec les plus jeunes » dont vient de témoigner dans Livres Hebdo Francis Geffard, se souvenant de ses années de très jeune libraire.

La responsabilité enfin est sans doute le trait le plus évident pour ceux qui ont travaillé sous ses ordres, c'est-à-dire avec lui. Jean Gattégno a eu la chance d'être devenu directeur à un moment idéal de l'histoire du ministère de la Culture, dont on rappelle classiquement qu'il fut marqué par la conjonction d'une effervescence créative, d'une volonté politique appuyée au plus haut niveau et de moyens budgétaires plus que confortables. J'ajouterai, ce que l'on dit moins souvent, qu'il était alors également facile - voire agréable - d'exercer le métier de fonctionnaire d’État. Ce dernier n'était pas en effet gangrené, comme il l'est devenu, par le transfert de ses missions à des opérateurs externes, la multiplication d'indicateurs autoréférentiels, le refus de toute prise de risque - en un mot, la peur panique des responsabilités. Écoutant les points de vue puis décidant, souvent assez vite, assumant ses choix et protégeant ses collaborateurs jusque dans leurs erreurs - quitte à les leur reprocher ensuite, hors de la présence des tiers -, il a laissé chez eux un modèle d'exercice de la fonction directoriale qui, trente ans plus tard, reste, pour moi au moins, insurpassé.

De ces trois qualités, ou plutôt de ces trois essences, il a su faire un usage magnifique (magique presque) comme directeur du livre et de la lecture. Pour résumer ces années, j’ai envie de reprendre la formule par laquelle, en juin 1999, Pierre Lepape avait rendu compte dans Le Monde du collectif Une voix qui manque: "Un fonctionnaire contre les bureaucrates ». Ces mots font écho au titre, un brin provocateur, Profession fonctionnaire, qu'avait choisi un autre grand serviteur de l'État, François BlochLainé, lorsque vers la fin des années 1970 il tirait un premier bilan de son parcours. 
Heureux dans l'État et ne craignant pas de l'affirmer, Bloch-Lainé s'inquiétait de voir se brouiller la relation entre le service du bien public et deux maîtres qu'à ses yeux le fonctionnaire ne pouvait pas ignorer, mais dont il devait se garder : l'argent et la politique. Deux tentations qui n'effleuraient pas Jean Gattégno dont la conception du métier de (haut) fonctionnaire obéissait, me semble-t-il, à une autre polarité : le droit d'un côté, l'autorité de l'État de l'autre.

Bien qu'énarque et ayant passé quatre ans au sein du ministère de l'Éducation nationale, j'étais bien peu juriste lorsque je rejoignis la direction du livre et de la lecture (DLL) le $1^{\text {er }}$ juin 1985 : j'avais appris ce qu'il fallait de droit administratif, de droit de la sécurité sociale et de droit budgétaire pour entrer à l'École nationale de l'administration (ENA); puis, dans mes fonctions à la Direction des bibliothèques, des musées et de l'information scientifique et technique (DBMIST), j'avais lu le Code des marchés publics et rédigé, tant bien que mal, les statuts de l'École des chartes et ceux de l'École centrale entre 1981 et 1984.

À la DLL, tout changea car la loi du 10 août 1985, dite « loi Lang », n'avait pas de meilleurs ennemis que les juristes - du juge d'instance de Bressuire aux experts du secrétariat général aux Affaires européennes, des avocats des centres Leclerc aux représentants de la Commission des Communautés européennes. Face à cela, la petite troupe des défenseurs du prix unique du livre était menée, du côté de l'État, par un normalien de la promotion Lettres 1956, professeur de littérature anglaise, Jean Gattégno, et, du côté du Syndicat national de l'édition, par un normalien de la promotion Lettres 1957, agrégé de lettres classiques, Pierre Frédet. Il est vrai que l'expérience syndicale de l'un, l'expérience administrative de l'autre les avaient aguerris en la matière.

Pour autant, je connais plus d'un universitaire fâché, au-delà du raisonnable, avec le droit - ce qui n'était évidemment pas le cas 
de Jean Gattégno. On n'a pas lieu de s'en étonner quand on sait qu'il fut l'introducteur en France de la Logique sans peine de Lewis Carroll et que droit et logique sont des disciplines proches, pas seulement formellement.

Jean Gattégno, nous nous en souvenons, avait belle prestance, de sorte qu'émanait de lui une autorité naturelle. Mais représenter, incarner même, comme cela sied à un directeur d'administration centrale, l'autorité de l'État, c'est tout autre chose : il est des hommes grands qui ne sont pas de grands fonctionnaires - il en est même beaucoup. Ce sont en effet des qualités intérieures qu'il faut, dans ce métier, mobiliser si l'on entend ne pas transiger avec ses responsabilités. Je place au premier rang de ces qualités le courage et la loyauté.

Le courage de Jean Gattégno, son collaborateur et ami Bernard Genton l'avait résumé par cette formule : "Je l'ai vu tenir tête à plus puissant que lui. » Au quotidien il se caractérisait, chez ce bretteur qui avait le goût de la formule et ne dédaignait pas la polémique, par une double attitude : appeler un chat un chat, aller à la bataille quand il fallait y aller.

La loyauté. On lui a reproché, stupidement, d'avoir servi le gouvernement de cohabitation de 1986-1988. Nous sommes ici plusieurs à pouvoir témoigner de la relation déférente et mutuellement respectueuse qu'il avait construite avec François Léotard, alors ministre de la Culture - les rapports étant tout autres avec le secrétaire d'État Philippe de Villiers. Comme l'a rappelé JeanSébastien Dupuit à Livres Hebdo, Jean Gattégno aurait sans l'ombre d'un doute quitté ses fonctions s'il avait senti l'essentiel remis en cause - ce ne fut pas le cas.

De cette alliance - courage et loyauté - qui fait à mes yeux le sens de l'État, je ne donnerai qu'une illustration. La scène a lieu à Rome, le 20 février 1986 - trois semaines avant les élections législatives en France -, à l'occasion d'une rencontre entre 
acteurs français et italiens de la " chaîne du livre ". Après une journée de travail, et avant le dîner, quelques toasts sont portés par les représentants des deux délégations : ambiance agréable, accrue par la chaleur de l'accueil romain et l'excellence des vins proposés - jusqu'à ce qu'un éditeur français (il s'agissait d'un écrivain, directeur littéraire d'une grande maison appartenant à un grand groupe) se livre à une attaque en règle du dispositif français de fixation du prix du livre : la législation liberticide portant le nom d'un ministre socialiste serait bientôt abrogée par le successeur, libéral, dudit ministre - dans son esprit, il aurait pu être lui-même ce successeur! Jean Gattégno se fâcha, rappela l'intéressé à un peu de dignité, les choses en restèrent là. On connaît la suite : ce n'est pas autour de la question du prix du livre que se déroulèrent les combats de la cohabitation, Jacques Chirac n'ayant pas besoin d'être convaincu des bienfaits du dispositif !

De manière presque trop symétrique, on peut opposer cette synthèse idéale, sur fond d'autonomie et de responsabilité, des années DLL - synthèse entre une personne, un monde (des mondes plutôt) professionnel(s) et un fonctionnement institutionnel - à la séquence suivante, autour de la Bibliothèque de France. Je laisse ici la parole à Pierre Lepape: "Les amis de Gattégno qui l'ont rencontré à l'époque où il se livrait à cette dernière bataille, avant celle contre la maladie et la mort, se souviennent de son grand rire brisé, de l'amertume qui voilait son éclat, de la colère et du dégoût qui durcissaient le timbre chaud de sa voix¹.»

Il faudra un jour, vingt-cinq ou trente ans après le témoignage à chaud livré par Jean Gattégno lui-même², écrire l'histoire,

\footnotetext{
1. Pierre Lepape, «Un fonctionnaire contre les bureaucrates », Le Monde, 18 juin 1999.

2. Jean Gattégno, La Bibliothèque de France à mi-parcours, de la TGB à la BN bis, Paris, Éditions du Cercle de la Librairie, 1992.
} 
à proprement parler politique, de ce projet, décrire les jeux d'acteurs et les modes opératoires... Je ne m'y essaierai pas ici, sinon pour rapporter ce qu'il me dit quelques semaines avant son éviction. J'étais passé le voir à son bureau de la place Valhubert, le 11 février 1992, juste avant la première réunion du jury du concours de conservateur de bibliothèque (qu'il présidait et que je vice-présidais). On était en pleine " affaire Habache », qui avait vu des hauts fonctionnaires - dont le secrétaire général du Quai d'Orsay, François Scheer - être démis de leurs fonctions afin de servir de fusibles à des ministres dont le président de la République n'entendait pas se séparer³. «Je ne serai pas le François Scheer de la Bibliothèque de France », me dit Jean Gattégno.

Il le fut, moins d'un mois plus tard. «Vous partez la tête haute, lui dit le ministre Biasini, et vous ne partirez pas n'importe où. » On sait ce qu'il en fut, et cette suite n'est pas drôle... Permettez-moi d'imaginer, pour éviter de rester sur cette note tragique, une utopie - celle par exemple qui aurait vu M. Biasini tenir sa promesse, et se montrer persuasif auprès du président de la République et de la Première ministre.

Nous sommes alors le 6 juin 2005. À l'occasion de ses 70 ans, nous invitons le conseiller d'État Jean Gattégno à une représentation, dans la grande salle du palais de Chaillot, du Julius Caesar - dont il connaît des pans entiers par cœur (« For Brutus is an honourable man ") - dans la formidable mise en scène de Deborah Warner"

Il est alors, deux ans après sa retraite, au milieu de son mandat de président de la commission d'avance sur recettes, et

\footnotetext{
3. L'affaire est rappelée et analysée par Olivier Beaud, La responsabilité des gouvernants, Paris, Éditions Descartes \& Cie, 1999.

4. Le programme de nos journées ne rendant pas compte de sa passion pour le cinéma et le théâtre, c'est ici l'occasion de la rappeler.
} 
travaille d'arrache-pied à la nouvelle édition des œuvres complètes de Dickens en Pléiade. Inutile de vous dire qu'il apprécie notre cadeau collectif : une caisse de Mission Haut-Brion comportant une bouteille de chacun des millésimes d'exception - 1981 à 1992 - qui nous ont vus travailler à ses côtés : nos plus belles années ! 


\section{Bibliothèques et développement de la culture}

par Gérald Grunberg

Pour introduire cette table ronde, je citerai une des premières interventions publiques de Jean alors qu'il venait d'être nommé Directeur du livre et de la lecture. C'était lors du colloque « Lectures et bibliothèques publiques » organisé à Hénin-Beaumont en novembre 1981. Il faut se remettre dans l'ambiance très particulière de ce moment : ce colloque qui se tient dans l'effervescence de l'après-mai 1981 rassemble tout ce que la France compte de bibliothécaires et d'acteurs de la diffusion du livre.

Jean commence par lire un court texte de Jack Lang qui déclare à l'assistance : «Deux maîtres mots résument notre politique : décentralisation et création... Pour la création, n'ayez aucun complexe. Nous savons, vous comme moi, qu'un tableau ne vit que d'être regardé, une musique d'être écoutée, un livre d'être lu. Le sort même de la création littéraire dépend de la démocratisation de la lecture. »

Puis il enchaîne et, comme très souvent, Jean parle pratiquement sans notes. On pourrait croire qu'il va se contenter de faire valoir le résultat du débat budgétaire qui vient d'avoir lieu au Parlement. En effet, le budget de l'État consacré au fonctionnement des bibliothèques municipales (BM) venait de passer de 17,5 millions de francs en 1981 à 214 millions en 1982, le budget d'équipement de 26 millions en 1981 à 115 millions, en même temps qu'une centaine d'emplois étaient créés dans les bibliothèques centrales de prêt (BCP), etc. Pour tous les bibliothécaires de France et de Navarre, c'était enfin le démarrage tant attendu d'une grande politique en faveur des bibliothèques 
publiques. Or Jean rappelle ces chiffres, ce qui fait la joie des bibliothécaires, mais il ajoute immédiatement que ce qui est au moins aussi important «c'est le contexte nouveau dans lequel se font ces choses et essentiellement, la volonté de décentralisation qui commande la politique de la Direction du livre et de la lecture ». Précisant que tout le monde, acteurs de terrain comme administration, va devoir opérer une véritable reconversion, ce sont ses termes, il développe quelques points particulièrement importants. D'abord, il annonce le changement de statut des personnels et l'égalité à venir entre fonctionnaires d'État et fonctionnaires territoriaux, ensuite il se prononce sur le fait qu'il faudra redéfinir les compétences entre les échelons et repenser l'Inspection, mais il précise : « je souhaite que nous continuions, nous, service national, à avoir un rôle d'appui technique à jouer pour les bibliothèques municipales, c'est-à-dire pour les collectivités élues qui ont une Bibliothèque et qui font travailler du personnel ». Enfin, il pose frontalement une question essentielle. Je cite :

«Il est certain que si on croit que décentraliser, cela veut simplement dire transférer un certain type d'organisation de Paris vers une région ou un département, on risque d'avoir de véritables déconvenues. La décentralisation dans l'optique du Gouvernement et pour le ministre de la Culture est une mesure d'autogestion.

Cela veut dire que le problème auquel nous risquons tous d'être confrontés est une mise en question de notre compétence. Ce que je veux dire, c'est qu'il vous faut, il nous faut, trouver des structures et une attitude qui permettent qu'à la base, là où vous travaillez, les décisions auxquelles on doit arriver dans tout service collectif soient prises non par celles et ceux qui ont la compétence, mais par l'ensemble de celles et ceux qui font fonctionner et utilisent le service public... En d'autres termes, on 
pourrait se poser la question de ce qui fonde notre légitimité ou de ce qui est notre légitimation. Je sais que dans le milieu des bibliothécaires comme dans le milieu des enseignants dont je sors, vous avez tendance à penser et nous avons toujours tendance à penser, dans nos professions respectives, que notre science légitime le pouvoir que nous exerçons et les modalités de ce pouvoir. Je suis de ceux qui pensent que pour les enseignants c'est extrêmement contestable. Peut-être que la plupart d'entre vous sont arrivés à la même conclusion dans l'univers des bibliothèques. Je n'en suis cependant pas sûr, et je ne suis surtout pas sûr que les élus et les lecteurs en aient été convaincus jusqu'à présent. Cela veut dire qu'il nous faudra trouver d'autres sources que la légitimation. Je me trompe peut-être mais il nous manquera comme Bible en quelque sorte, notre compétence scientifique. Nous ne serons plus, vous ne serez plus, les représentants de l'État dans les BM et, vraisemblablement un jour, dans les BCP. Sur quoi alors se fondera notre pouvoir, votre autorité ? Je ne veux pas dire le pouvoir d'oppression mais le pouvoir d'organiser votre établissement. C'est une interrogation, je n'ai pas de réponse.

En dernier lieu, j'ai entendu dire, et je comprends cette inquiétude, que pour beaucoup de professionnels, l'un des risques de la décentralisation vient de ce que les élus locaux sont souvent mal informés, voire peu concernés par le problème de la lecture. On retrouve là le problème de la compétence. Il est probable que nous sommes globalement plus compétents pour parler des bibliothèques que tel ou tel élu. Il n'est pas évident du tout que nous soyons plus compétents pour parler de la lecture.

La décentralisation est un enjeu non pas technique mais politique. Si nous ne la prenons pas ainsi, nous la raterons et tant que je serai Directeur du livre et de la lecture, je m'arrangerai pour qu'on la prenne de manière politique. » 
Quelle formidable profession de foi qui vise un double objectif : affirmer la dimension politique de la question de la lecture publique, mettre en mouvement toute une profession en ébranlant ses fondements corporatistes, et ne pas craindre d'en appeler à l'utopie, l'autogestion, pour créer une dynamique.

Quelle honnêteté en même temps. Il en faut pour accepter de n'être pas celui qui apporte toutes les réponses et pour le dire publiquement, illustrant par cette façon d'aller à contre-courant que l'autorité peut s'exercer autrement que sous la forme du sujet supposé savoir. C'est ainsi qu'il s'adressait aux bibliothécaires de lecture publique et c'est avec le même souci d'authenticité qu'il exercera sa fonction de délégué scientifique dans la conception et la réalisation de la future Bibliothèque nationale de France (BnF).

Enfin, ce qui frappe dans ce discours, hautement politique, c'est qu'il est habité par une vision. Vision que l'histoire va d'ailleurs confirmer sur bien des points, même lorsque c'est d'une façon totalement imprévisible, je pense par exemple au rôle joué par Internet dans le rapprochement entre bibliothécaires et lecteurs. Je souligne cette dimension car c'est peut-être aussi pour cela que nous manque Jean : il était la figure trop rare d'un haut fonctionnaire qui ne s'est jamais contenté d'être un simple rouage dans cette machine si bien huilée qu'est l'administration.

Du texte que j'ai cité, on pourrait, me semble-t-il, revenir sur quelques aspects pour illustrer l'actualité de la pensée de Jean, mesurer le chemin parcouru, se demander ce qui est dépassé et ce qui ne l'est pas et, au fond, apprécier comment nous avons fait ou non fructifier l'héritage.

Pour ma part, je retiens plusieurs thèmes :

$\odot$ Continuité et rupture. Seuls les plus anciens se souviennent de ce qu'était la misère des bibliothèques publiques françaises 
avant 1981. Un rapport, commandé juste avant les élections par le prédécesseur de Jean, en donnait toute la mesure et dressait en même temps un plan très conséquent pour résorber ce retard. Ce rapport volumineux avait été rédigé par Louis Yvert, chef du service des bibliothèques à la DLL, et son équipe. Certains pressaient Jean de mettre ce rapport à la poubelle et d'attendre les conclusions du rapport qui venait d'être commandé par le nouveau ministre à Bernard Pingaud et Jean-Claude Barreau. Il n'en fit rien et, pour préparer le budget 1982 qui vit le budget de la lecture publique être multiplié par dix, il s'appuya largement sur le plan de rattrapage proposé par Louis Yvert. Ce plan proposait pour l'essentiel de développer les structures existantes : BM et BCP. Le rapport Pingaud-Barreau faisait le même constat concernant la misère des bibliothèques françaises, mais les propositions étaient pour partie différentes, notamment la construction de médiathèques régionales et la mise en place de services régionaux de coopération qui ne virent pas le jour, en tout cas pas sous la forme préconisée.

Et de fait, si le retard a globalement bien été rattrapé dans les villes et les départements, la construction d'un réseau reliant les différents échelons, municipal (BM), départemental (BCP devenues bibliothèques départementales de prêt [BDP]), régional, national (BnF), n'a pas connu le même succès. Alors que s'ouvre une nouvelle phase de décentralisation qui va mettre en avant les régions et redéfinir les compétences des différents échelons territoriaux, cette question revient en pleine lumière. Elle ne saurait se poser aujourd'hui sans envisager toutes les catégories de bibliothèques, $\mathrm{y}$ compris les bibliothèques universitaires. On se souvient que Jean Gattégno avait souhaité avec Denis Varloot, alors responsable des bibliothèques universitaires, créer cette réflexion globale qu'il encouragera également lors de la création de la future BnF. Que faut-il en penser aujourd'hui? 
$\odot$ Maintien du rôle de l'État. Jean sait que l'État doit accompagner l'effort de rattrapage et, tout convaincu qu'il soit du bienfondé de la décentralisation, il ne ménagera pas sa peine pour faire en sorte que soit institué en faveur des BM un régime dérogatoire au droit commun de la décentralisation. C’est le fameux concours particulier au bilan plus que positif. Quid aujourd'hui de ce dispositif qui devait au départ être transitoire ? Au-delà de ce dispositif, de la décentralisation des BCP, des tentatives de faire évoluer le régime des BM classées, etc., existe-t-il encore une question de décentralisation en matière de bibliothèques et de lecture?

$\odot$ Le rôle des élus. On l'a vu dans le texte que j'ai cité, Jean avait un immense respect pour les élus dont il estimait que la légitimité était très supérieure, du fait du suffrage universel, à celle que confère le savoir professionnel (faut-il y voir l'influence de Debord et de sa critique des experts ?). Comment se sont finalement calés dans le temps les rapports entre bibliothécaires et élus ? Jean ne se contentait pas d'appeler au respect des élus, il a multiplié les démarches pour les écouter et les associer à la mise en œuvre de la politique du livre et de la lecture. Non seulement il répondait à toutes les invitations d'élus, dans la plus grande comme dans la plus petite des communes, mais il fit également plusieurs tentatives pour mettre en place des structures de concertation permanente. Quelle forme cela pourrait-il prendre aujourd'hui ?

$\odot$ L'évolution du métier de bibliothécaire. On imagine mal aujourd'hui le choc que fut pour une bonne partie de la profession le discours d'Hénin Beaumont, son appel à redéfinir la légitimité du bibliothécaire, son exhortation à inventer de nouvelles pratiques qui associent les lecteurs (on dirait aujourd'hui les usagers...). C'est la même conviction qui l'anima envers les chercheurs et les universitaires lorsqu'il s'est agi de définir la 
future $\mathrm{BnF}$. Au-delà des avancées contingentes que permet le Web participatif, qu'en est-il aujourd'hui de la volonté politique sur ce point?

J'aimerais pour terminer cette introduction évoquer encore deux aspects qui ne sont pas directement traités dans le discours d’Hénin Beaumont.

Le premier concerne le rapport de Jean, homme de culture s'il en fut qui jamais n'opposa modernité et classicisme, à l'importance qu'il convenait d'accorder à la création architecturale pour les bâtiments de bibliothèques. C'est véritablement sous son impulsion que fut dépassée, non sans peine, la vulgate très répandue qui voulait que pour être fonctionnel un bâtiment devait le plus possible ressembler à une boîte à chaussures. Une génération de $\mathrm{BM}$ et de BCP a vu le jour qui a contribué à la création architecturale dans notre pays. En retour, la qualité architecturale de ces équipements a contribué à en modifier l'image. Comment évaluer aujourd'hui cette dimension?

Le second regarde la question de la lecture dans son ensemble. Jean a essayé avec la générosité qui le caractérisait de favoriser l'approche la plus englobante possible, faisant une place réelle à ce que l'on appelait le "tiers réseau » : bibliothèques de comités d'entreprise, bibliothèques de prisons et d'hôpitaux, bibliothèques de rue, etc. Jamais les animateurs de ces bibliothèques n'ont autant été regardés comme partenaires à part entière. Que sont-ils devenus? En fait, nous voyons de nouvelles formes de solidarité par la lecture émerger, à côté, en dehors, des politiques publiques. En d'autres termes, de quoi Bibliothèques sans frontières, qui fait au demeurant un travail admirable, est-elle aujourd'hui le nom?

Pour illustrer cette introduction mais surtout la corriger, la compléter et apporter des éléments de réponses aux questions posées: Valérie Tesnière, Denis Llavori, Nicolas Galaud vont apporter leurs éclairages. 


\section{L'engagement scientifique de Jean Gattégno dans les bibliothèques}

par Valérie Tesnière

Le nom de Jean Gattégno, Directeur du livre et de la lecture de 1981 à 1989, reste attaché au développement de la lecture publique, secteur auquel il donne une impulsion décisive dans le contexte politiquement favorable du ministère de Jack Lang. Son engagement dans l'action publique culturelle est plus marqué par son parcours d'universitaire qu'on ne le pense, et ce, non seulement parce qu'il a été un grand traducteur de la littérature anglaise, mais aussi par son engagement personnel dans la formation des étudiants à l'université de Vincennes. Après la Direction du livre, il devient délégué scientifique de l'Établissement public de la Bibliothèque de France (EPBF), poste qui le passionne, car il y voit un prolongement de son action et un enjeu essentiel de modernisation de la recherche et de l'accès au savoir à travers un établissement phare. Ce fil rouge, fait de convictions scientifiques autant que politiques, l'a guidé de la Direction du livre et de la lecture à la direction scientifique de l'EPBF.

\section{Lecture publique?}

Le Directeur du livre qui prend ses fonctions en 1981 n'établit pas d'échelle de valeur : lire un roman peut être une forme de joie ou de bonheur, étudier et chercher une autre. D'ailleurs, pour cet excellent connaisseur du $\mathrm{xIX}^{\mathrm{e}}$ siècle, la fiction peut magistralement éclairer la compréhension du passé ou du présent. La lecture publique, en bref, s'applique à toutes les formes de lecture. 
C'est un service public, qui concerne aussi bien l'apprentissage des savoirs que l'éveil à la culture.

Une telle conception n'allait pas de soi en 1980. La séparation institutionnelle entre bibliothèques de lecture publique et bibliothèques universitaires et de recherche pèse alors de tout son poids. Les militants des associations professionnelles sont obnubilés par le retard français en matière de promotion de la lecture comparé aux équipements des pays d'Europe du Nord ou des États-Unis. Au sein de l'Association des bibliothécaires français $(\mathrm{ABF})$, les représentants des bibliothèques territoriales sont majoritaires, les conservateurs exerçant dans les bibliothèques de recherche sont quasi absents et ceux de la Bibliothèque nationale (BN) sont davantage impliqués dans les instances internationales (International Federation of Library Associations and Institutions [IFLA]) que dans les instances nationales. La profession est peu unie. Ses divisions sont anciennes. À l'ABF, la fonction d'étude et de recherche continue d'être synonyme d'une érudition dépréciée et les bibliothèques universitaires sont perçues comme des services documentaires voués à gérer les conséquences du passage à l'université de masse. Les points communs de ces dernières avec les structures rattachées au ministère de la Culture semblent limités. En 1981, Jean Gattégno découvre une profession de bibliothécaire encore clivée par les combats des années 1970 en faveur de la promotion d'une lecture publique au profit quasi exclusif des bibliothèques municipales ou des bibliothèques centrales de prêt (Noë Richter, Michel Bouvy), assez loin des milieux d'universitaires, d'écrivains ou de militants politiques qu'il a l'habitude de fréquenter.

Dès sa nomination, il épouse la cause de la promotion de la lecture avec enthousiasme. Lecture et non lecture publique au sens où l'entendent les professionnels des bibliothèques. Si publique il y a, c'est dans le sens d'un accès libre et ouvert au plus grand nombre, en particulier ceux pour lesquels cet accès est difficile 
ou malaisé (prisons et hôpitaux) ou ceux qui se désintéressent de la lecture (jeunes, c'est-à-dire adolescents, pour lesquels il crée une mission spécifique). Le développement de la lecture est un enjeu politique qui n'est pas l'apanage de l'État, même si ce dernier doit réinvestir cette mission beaucoup plus qu'il ne l'a fait jusqu'alors, en s'appuyant sur les institutions comme sur les associations. C'est à cette condition qu'il devient possible de parler de lecture publique. L'intérêt que Jean Gattégno portait aux associations, pourvu que celles-ci concourent sans sectarisme à cette finalité émancipatrice, s'est manifesté dans le soutien qu'il a apporté, par exemple, à la Bibliothèque des amis de l'instruction, dans le $3^{\mathrm{e}}$ arrondissement de Paris, en place depuis le XIX ${ }^{\mathrm{e}}$ siècle. Cette anecdote n'est pas qu'un clin d'œil à Dickens. Les « amis de l'instruction » doivent être encouragés au même titre que des institutions ayant pignon sur rue. Il est en effet tout aussi légitime de lire des romans, le journal que des essais ou des manuels, au sein de structures qui œuvrent dans la même direction.

Cette attitude très ouverte a pu susciter parfois de l'incompréhension tant était ancrée dans les associations professionnelles l'idée que les missions de l'État réaffirmées depuis la Libération et symbolisées par les BCP ne pouvaient être exercées par d'autres acteurs que des professionnels dûment formés. Le jacobinisme culturel va être ébranlé dès 1981 par la mise en œuvre de la décentralisation qui transforme les bibliothèques dites « centrales de prêt » en bibliothèques départementales de prêt. Dans une lecture très politique de la réforme, Jean Gattégno réaffirme le primat du politique et le rôle des élus des collectivités territoriales dans le développement de la lecture publique. En cela comme dans son appui aux associations, il a pu se heurter aux professionnels des bibliothèques. Il leur a surtout fait franchir un cap en leur donnant à comprendre que la diversité de pratiques de la lecture renvoie à une diversité d'acteurs, dont la légitimité est à apprécier en fonction de la philosophie qui les anime plus 
qu'en fonction des institutions auxquelles ils se rattachent. Cette philosophie correspond à la haute idée qu'il a d'une éducation par la lecture, quels qu'en soient les vecteurs.

\section{Usages de la lecture}

En insistant sur la diversité de pratiques de lecture, on aborde une problématique émergente au milieu des années 1980, celle des usages de la lecture. Quand Jean Gattégno prend son poste de délégué scientifique à l'EPBF en novembre 1989, la notion d'« usages de la lecture » ne s'est pas imposée comme aujourd'hui parmi les professionnels des bibliothèques ou les cercles universitaires. Les sociologues, analystes des pratiques culturelles, utilisent alors les outils caractérisant leur discipline, des grilles par catégories socioprofessionnelles (CSP), pour déterminer le taux de pénétration de la lecture. Le contenu des lectures, la manière dont les gens lisent et les supports qu'ils privilégient, tout ceci est au second plan. De nombreuses études s'intéressent notamment à l'illettrisme, préoccupation pertinente du point de vue des politiques publiques, notamment de l'éducation, tandis que l'on observe avec dépit que la majorité des " gros liseurs » relève des catégories CSP+. Les promoteurs du développement de la lecture sont déroutés par ces constats quantitatifs et s'en servent dans un premier temps pour plaider leur cause. La presse se fait l'écho de ces enquêtes auprès du public; les sociologues et les journalistes qui font autorité sont alors assez loin dans l'ensemble des recherches en cours dans les domaines des sciences cognitives, des sciences de l'information, voire de l'histoire de la lecture, qui insistent sur la diversité des usages de la lecture, dont le livre est un support parmi beaucoup d'autres. L'intérêt de ces travaux apparaîtra au grand jour avec la révolution numérique, qui apporte un nouveau regard sur enjeux des pratiques de lecture et d'appropriation des connaissances. 
Bousculant la relation au livre en tant que codex imprimé, Internet a en effet beaucoup contribué à désacraliser celui-ci, en tant que réceptacle d'une lecture suivie, et à généraliser la prise de conscience de la simultanéité de différentes formes de lecture chez un même individu: fragmentées, au long cours, rapides, lentes, avec annotations, etc. Il convient à cet égard de parler du numérique, Internet étant le vecteur de dissémination de ces pratiques nouvelles plus que leur origine. La lecture fragmentée n'est pas une nouveauté : la recherche d'une information dans une base de données ou dans un journal se pratique quotidiennement. Le numérique démultiplie les modes d'accès et de navigation dans les textes et bouscule la hiérarchie symbolique des supports imprimés, où le livre l'emporte sur le journal ou la documentation. Ainsi la lecture annotée, qui intéresse alors surtout les chercheurs en lettres et sciences humaines car l'outil numérique facilite la recherche d'occurrences dans les textes eux-mêmes et en renouvelle radicalement les modes d'appréhension. Au début des années 1990, Internet est encore dans les limbes et n'est pas entré dans la vie quotidienne. La lecture annotée par ordinateur en est à ses débuts. Jean Gattégno, désormais délégué scientifique de l'EPBF, s'y investit avec enthousiasme, en apportant un soutien public au prototype du poste de lecture assistée par ordinateur (PLA0). Il en est luimême un expérimentateur très investi en tant que traducteur.

En quoi ces réflexions développées au sein de l'EPBF comme dans d'autres laboratoires de recherche ont-elles eu une incidence sur le projet scientifique général, porté par Jean Gattégno ${ }^{5}$ ? Les problématiques de la lecture sont connues du délégué scientifique. S'y ajoute une connaissance approfondie des bibliothèques étrangères, de la British Library à la reference library américaine et d'autres grandes bibliothèques de recherche, européennes ou américaines. 
Ces points de comparaison permettent de proposer une organisation des espaces de lecture conforme à l'exigence démocratique du projet présidentiel. Deux niveaux seront accessibles au public. L'articulation du Haut-de-jardin et du Rez-de-jardin de la future Bibliothèque de France se fonde sur une analyse des usages et non exclusivement sur une partition des lecteurs selon des critères socioprofessionnels préétablis. Une même personne dotée d'un capital intellectuel donné peut éprouver le besoin de mixer des usages multiples de la lecture, par exemple une démarche d'approfondissement dans sa spécialité à une démarche de mise à niveau ou d'information dans un domaine proche du sien, sans même parler de l'envie de lire quelque chose de très différent encore aux antipodes de ses centres d'intérêt principaux. Elle peut être conduite à fréquenter un premier niveau de référence tout en consultant dans un second temps dans la bibliothèque de recherche des documents plus spécialisés. On peut appliquer ce schéma à toutes sortes d'individus relevant de catégories socioprofessionnelles diverses, avec des niveaux inégaux de formation : certes un chercheur, un étudiant, mais aussi un ingénieur, un chômeur en reconversion, un amateur d'histoire, etc.

Cela n'allait alors pas de soi. Ni pour les sociologues, ni pour les politiques qui s'appuient sur les travaux de ceux-ci, ni pour un certain nombre d'intellectuels. Le concept de «bibliothèque publique de recherche », correspondant à une première appropriation de la recherche au niveau Haut-de-jardin, n’a pas été bien reçu. Au sein de l'équipe scientifique réunie autour de Jean Gattégno, c'est ainsi qu'on avait tenté de définir le niveau « grand public » de la future BnF, signifiant par là l'intérêt et la nécessité d'une « propédeutique de la recherche ", d'un apprentissage démocratique des méthodes de la recherche. Reference library: le concept anglo-saxon ne passait pas davantage dans une "République des lettres » française, qui ne s'était pas encore remise des conséquences de la démocratisation de l'enseignement supérieur et qui était au fond soucieuse 
de ne pas trop mélanger dans un même espace de travail les professeurs ou les étudiants ou de simples citoyens, dont les comportements pourraient se révéler incompatibles. Parmi les universitaires mobilisés autour de Jean Gattégno, il s'en est pourtant trouvé un certain nombre pour considérer que le Haut-de-jardin avait une utile fonction d'information et de référence qui les conduirait à fréquenter ce niveau, avant de rejoindre le niveau du Rez-de-jardin où seraient communiqués les fonds plus rares ou plus "pointus ", anciens ou issus du dépôt légal, conservés en magasin ${ }^{6}$. C'est donc l'usager qui, en fonction de l'étape de sa recherche de documentation, irait d'un niveau à l'autre. Le statut de la personne ne serait pas le seul déterminant pour définir son inscription privilégiée à l'un des deux niveaux. On ne s'appesantira pas sur la polémique qui discrédita une partie du travail produit par les équipes et les groupes de travail mobilisés par Jean Gattégno. Le rapport d’André Miquel sur la misère des bibliothèques universitaires ${ }^{7} \mathrm{a}$ pu faire de surcroît redouter une invasion des salles par des foules d'étudiants : il contribua probablement à brouiller le message. Le plan Université 2000 permit ultérieurement à l'État, avec l'appui de la région Ile-de-France, de rendre cette crainte largement infondée. Cet exemple illustre que la démocratisation de la lecture est un combat à multiples dimensions. Lecture publique, outils au service de la recherche, il y a bien en somme des points communs.

\section{Encyclopédisme, pluridisciplinarité, interdisciplinarité}

Portée par cette exigence de démocratisation de l'accès au savoir, la démarche du délégué scientifique de l'EPBF consista à mobiliser un nombre considérable d'universitaires et de professionnels

\footnotetext{
6. Notamment François Dagognet, Jacques Brunschwig ou Immanuel Wallerstein parmi les philosophes.

7. André Miquel a été administrateur de la BN de 1984 à 1987. Le rapport sort en 1989.
} 
des bibliothèques au sein de groupes de travail définissant les contours de la future grande bibliothèque. La question des usages de la bibliothèque rejoint ici l'enjeu de ses contenus. Le projet de Très Grande Bibliothèque (TGB) fait le pont entre un phantasme des bibliothèques, l'actualité de l'encyclopédisme des Lumières, et des enjeux scientifiques du temps présent. Le savoir contemporain, incarné dans une nouvelle bibliothèque, ne pouvait pas en effet couper les humanités des sciences sociales, ni des sciences. Cet aspect, sans doute plus méconnu, mérite qu'on s'y attarde. En effet, la place de certaines disciplines au sein de la future bibliothèque a suscité un vif débat. Tout particulièrement celle des sciences et techniques, dont un certain nombre d'historiens en particulier ne voyaient pas l'intérêt dans le nouveau temple du savoir. L'argument invoqué, non sans fondement, était que les chercheurs en sciences utilisent depuis longtemps des outils accessibles depuis leur laboratoire. Avec le recul, dans cette réaction, on peut aussi déceler une défiance forte vis-à-vis de la culture scientifique de la part des sciences humaines.

Car les premiers à se mobiliser pour soutenir le projet présidentiel ont été des chercheurs en sciences " dures ", sans doute parce que l'enjeu politique d'une diffusion de la culture scientifique dans la société est pour eux fondamental, et parce que nombre d'entre eux éprouvent le besoin de sortir de leur spécialité pour la raccorder à d'autres problématiques, notamment d'ordre sociétal. Cela va toutefois plus loin. « [...] Il est clair qu'il n'existe pour aucun scientifique ou lecteur scientifiquement formé de centre permettant de prendre connaissance de l'état d'une problématique scientifique dans une discipline voisine de la sienne. C'est donc à ce niveau référentiel que la Bibliothèque de France devrait se situer pour drainer un public souhaitant appréhender l'évolution de la science ${ }^{8}$. » Cette réflexion est directement issue des discussions du groupe de travail «sciences »

8. Jean Gattégno, La Bibliothèque de France à mi-parcours, op. cit., p. 169. 
auquel participaient des mathématiciens comme Pierre Barral, Jean-Pierre Bourguignon et les animateurs du réseau Mathdoc, le physicien Franck Laloë et bien d'autres ${ }^{9}$. En sciences, on ne se limite toutefois pas à la définition d'un niveau (référence ou recherche); la place des sciences au sein d'un plus vaste ensemble est débattue.

Jean Gattégno vient d'un univers scientifique différent des habitués de la salle Labrouste, majoritairement issus des universités de lettres et sciences humaines. Il a fréquenté la salle Labrouste de la BN mais aussi la Bristish Library, qui n'exclut des humanités ni les sciences, ni le droit ou l'économie. À l'université de Vincennes, où il a enseigné, les débats sur les enjeux de la pluridisciplinarité ou de l'interdisciplinarité ont porté bien des renouvellements de l'université française après 1968. De plus, ses engagements personnels l'ont sensibilisé aux projets pédagogiques innovants s'appuyant sur la lecture. Fort de cette expérience, il anime avec conviction le travail des commissions d'acquisitions destinées à définir la politique documentaire. On tente de cerner les disciplines en mutation, utiles aux champs des sciences humaines et sociales: "L'histoire des sciences - dotée à la Bibliothèque nationale de riches collections qui n'ont d'ailleurs pas cessé d'être enrichies - mais aussi la documentation concernant les disciplines transverses : études sur la science ${ }^{10}$, sciences cognitives, biotechnologies, pourraient constituer le socle d'un futur département des sciences ", écrit Jean Gattégno"11. La forte mobilisation des groupes de travail est ainsi venue de la conviction partagée par une majorité des universitaires alors sollicités qu'on ne pouvait, avec un projet d'une telle envergure, laisser de côté des champs en complète évolution. Ce sont essentiellement

\footnotetext{
9. C'est ici l'occasion de saluer le travail de Francine Masson, alors directrice de la bibliothèque de l'École polytechnique après avoir exercé en lecture publique.

10. Traduction française du concept de science studies.

11. Jean Gattégno, La Bibliothèque de France à mi-parcours, op. cit.
} 
les biologistes et les médecins qui sont restés à l'écart de ce grand brassage d'idées nourri par les différentes commissions d'acquisitions. Ont répondu présents les philosophes et les mathématiciens déjà cités, ainsi que ceux impliqués dans le renouvellement des sciences sociales dans une approche pluridisciplinaire, et, pour les sciences, dans les enjeux d'interdisciplinarité. La place des sciences cognitives, aujourd'hui reconnue, a ainsi engendré des débats assez vifs ; la mise en cause de leur légitimité débordait largement le champ de la psychanalyse et interroge d'ailleurs sur des blocages plus généraux d'ordre épistémologique quant à la réception de la recherche interdisciplinaire.

Pour s'en tenir au registre des sciences sociales, les chercheurs en économie ou politique exprimèrent aussi leur intérêt pour les enjeux documentaires de la TGB, entre autres afin d'exploiter des sources abondantes en provenance du dépôt légal (statistiques, presse technique...), au même titre que les archives publiques. Le cas de la linguistique, discipline totalement renouvelée au cours $\mathrm{du} \mathrm{xx}^{\mathrm{e}}$ siècle, montrait aussi que la production scientifique étrangère qui ne relevait pas de la tradition philologique avait été inégalement couverte par la veille documentaire assurée à la $\mathrm{BN}^{12}$. Dotée de moyens insuffisants, la politique d'acquisitions de cette dernière n'avait pas pu suivre, sauf dans des domaines circonscrits, portés par un noyau d'universitaires français, essentiellement historiens et littéraires. L'exemple de la linguistique révélait là aussi un malaise plus profond : un établissement national, dont la mission est d'accueillir largement au-delà d'une institution universitaire donnée, peut-il se tenir à l'écart des évolutions épistémologiques au cœur même des humanités ? Sans vouloir tout embrasser de façon exhaustive, ce qui d'ailleurs n'est pas la définition de l'encyclopédisme, le travail des commissions a

12. Christian Puech et Valérie Tesnière, «Expertise scientifique et évaluation des collections, une méthode appliquée aux fonds de linguistique de la $\mathrm{BnF}$ », Bulletin des bibliothèques de France, 2000, $\mathrm{n}^{\circ} 4$. 
fait remonter la nécessité de mettre en phase l'institution avec les enjeux scientifiques de la recherche, débattus au niveau international ${ }^{13}$.

De façon sous-jacente se croisent au fond sans cesse des inquiétudes de divers ordres chez les intellectuels les plus hostiles au projet : d'une part, des réticences vis-à-vis d'acquis relativement récents, ce qui coïncide d'ailleurs avec le début d'un reflux éditorial dans les sciences humaines françaises ${ }^{14}$; d'autre part, l'enjeu de partage du savoir au-delà de l'environnement immédiat de la communauté de chercheurs avec lesquels on est en relation. Enfin, la nécessité pour la BN de s'intégrer davantage au réseau des bibliothèques françaises de recherche, qu'elles relèvent de la Culture ou de l'Enseignement supérieur, avait la même signification. D'une certaine façon, le développement d'Internet accentuera radicalement la démocratisation, qui est déjà en débat avec la Bibliothèque de France.

C'est bien d'un engagement politique dont il s'agit tout au long de ce parcours, dont le fil conducteur est celui d'une émancipation par la lecture, condition de l'accès au savoir. Héritage des Lumières, sans nul doute, mais aussi questionnement très contemporain. Il ne faut pas s'y tromper. L'encyclopédisme tel qu'il se définit pour la BnF au tournant du xxi ${ }^{e}$ siècle n'est plus le même que deux siècles auparavant. C'est un choix documentaire raisonné et non une course à l'exhaustivité. Il convoque dans un registre pluriel des disciplines différentes. Il s'inscrit aussi dans un registre interdisciplinaire dans la mesure où il piste et met en valeur le renouveau de la recherche, à l'affût de ce qui bouge, à l'échelle des moyens d'une institution nationale qui se doit d'être

13. Valérie Tesnière, «L'ouverture du site François Mitterrand. Mutations, enjeux scientifiques et organisation du travail », Jacqueline Sanson, une vie au service de la BnF, p. 57-65.

14. Notamment pour les travaux des historiens. Voir Sophie Barluet, Édition de sciences humaines et sociales : le cœur en danger : rapport de mission pour le Centre national du livre sur l'édition de sciences humaines et sociales en France, mars 2004. 
largement ouverte. Jean Gattégno a su porter une telle ambition à la hauteur des enjeux de démocratisation des connaissances, dans la continuité de son parcours personnel autant que public. Les réalisations tant en matière de politique de la lecture qu'en politique scientifique de la $\mathrm{BnF}$ ont confirmé la justesse de son combat. 


\section{Vingt ans après}

par Nicolas Galaud

Je n'ai jamais rencontré Jean Gattégno. Je suis entré dans le monde des bibliothèques, alors qu'il avait quitté ses fonctions de Directeur du livre et de la lecture pour celles de délégué scientifique de l'EPBF. Je n'ai donc pas de raison personnelle d'être nostalgique de cet âge d'or culturel que fut le premier septennat mitterrandien, dont je n'ai reçu les bienfaits qu'indirectement. Sur le plan professionnel en revanche, j'ai bien conscience de l'importance de cette période pour le développement des bibliothèques françaises, non seulement par l'impulsion donnée à la construction de nouveaux équipements, mais aussi parce que beaucoup des dispositifs inventés à cette époque continuent de servir de cadre à notre travail quotidien. Évoquer Jean Gattégno aujourd'hui, c'est nécessairement poser la question du rôle de l'État en matière de lecture publique. Si l'État aujourd'hui est plus un accompagnateur qu'un stratège, il continue d'occuper une place importante dans le domaine des bibliothèques aux côtés des collectivités territoriales. Cette place est-elle appelée à évoluer, notamment à l'occasion de la réforme territoriale qui s'annonce ? L'État doit-il encore se mêler de cette question?

Il est incontestable que les bibliothèques françaises, tous types confondus, ont connu un développement remarquable ces trente dernières années. Le retard français en la matière a été largement comblé. Notre pays peut même apparaître, sur certains points, comme relativement exemplaire. Pour ce qui est des bibliothèques des collectivités territoriales, ce développement est dû à une ambition partagée, à une volonté conjointe des élus locaux et du ministère de la Culture. 
Ce mouvement de modernisation, initié dans les années 1970, s'est considérablement amplifié à partir de 1981 sous l'effet conjugué d'un volontarisme politique affirmé de la part de l'État, de l'augmentation conséquente des moyens alloués au ministère de la Culture et d'une dynamique nouvelle engendrée par les lois de décentralisation de 1982-1983. Dans le domaine culturel à vrai dire, les compétences étaient déjà largement partagées entre l'État et les collectivités locales - les BM par exemple sont de longue date à la charge des communes - et la territorialisation de l'action culturelle déjà engagée, encadrée par de nombreux dispositifs contractuels. Lors de l'acte I de la décentralisation institutionnelle, les transferts en matière culturelle ont été, on le sait, limités. Ils ont concerné les archives départementales, déjà largement à la charge des départements, et bien évidemment les BCP.

Le renforcement du rôle des collectivités territoriales en matière de culture tient moins à des dispositifs de transfert de compétences, qu'à la liberté d'action nouvelle dont elles ont disposé. Comme l'a rappelé récemment Guy Saez : "c'est moins l'effet direct de la loi que le contexte qu'elle crée qui explique l'espace de liberté que les élus locaux vont s'approprier. La décentralisation a fait de la culture un attribut nécessaire de la puissance publique locale et de la légitimité de tout aspirant ou détenteur de pouvoir politique ${ }^{15} »$. Les bibliothèques ont largement profité de ce mouvement, devenant sur tout le territoire des équipements culturels de base, parfois le seul dans de nombreuses communes, au service de multiples politiques publiques locales.

Plusieurs points sont à souligner ici. Tout d'abord, cet effort de modernisation s'est produit en dehors de toute obligation réglementaire. Dans le domaine de la lecture publique, comme

15. Guy Saez, «Le Roman de la décentralisation », L'Observatoire, la revue des politiques culturelles, hiver 2013, n 43, p. 13. 
dans d'autres secteurs culturels, les collectivités territoriales sont libres d'agir ou de ne rien faire, sans risque de sanction, sinon celle, plus ou moins hypothétique, du suffrage universel. En second lieu, l'action de l'État peut être jugée positivement, ce qui n'est pas forcément le cas dans tous les domaines de la culture, en raison notamment du caractère très incitatif des aides qu'il apporte aux communes et aux départements, par le biais du concours particulier de la Dotation générale de décentralisation (DGD). Il faut noter enfin la constance dans le temps de l'engagement de l'État, qui a manifesté régulièrement sa volonté d'agir aux côtés des collectivités territoriales en faveur du développement de la lecture, au-delà des alternances politiques. Les outils, les dispositifs et les principes qui fondent l'action de l'État dans le domaine du livre et de la lecture, dont la plupart ont été mis en place à l'époque de Jean Gattégno, à commencer par le concours particulier déjà cité, s'ils ont connu depuis des ajustements techniques, sont restés à peu près les mêmes aujourd'hui encore.

L'environnement national et international a pourtant singulièrement changé depuis trente ans. Les politiques culturelles sont de plus en plus territorialisées, les collectivités assument une part prépondérante du financement public de la culture, l'intercommunalité progresse, même si cette progression est plus lente en matière culturelle que dans d'autres domaines, la métropolisation est un phénomène majeur qui structure fortement le secteur culturel et va s'amplifier avec les recompositions territoriales en cours. Plus largement, la révolution numérique et le poids croissant des industries culturelles dans un contexte de globalisation remettent en question certains des principes qui fondent notre modèle culturel français.

On peut interpréter la permanence des modalités de l'action de l'État dans le domaine de la lecture publique de plusieurs manières. Certains pourraient y voir l'expression d'une incapacité de sa 
part à repenser ses missions, son organisation et son fonctionnement, la preuve du passage d'un ministère animé par « l'esprit de mission " et le militantisme, à une " administration de gestion », pour reprendre une expression de Philippe Poirrier ${ }^{16}$, administration davantage préoccupée par la pérennisation de ses moyens d'action et de son périmètre d'intervention qu'animée par une vision prospective et la volonté d'impulser des politiques nouvelles. Il est vrai que le contexte actuel, marqué par la crise des finances publiques et le recul de la culture dans l'ordre des priorités gouvernementales, rend toute velléité de réforme en profondeur plus compliquée, quand elle n'est pas guidée, comme la révision générale des politiques publiques (RGPP) et ses avatars, par des logiques purement comptables. Par ailleurs, l'autonomie croissante des grands établissements nationaux, le plus souvent parisiens, leur poids budgétaire de plus en plus élevé et, s'agissant du livre, la séparation organique de la Direction du livre et du CNL sont autant d'éléments qui ont limité ces dernières années les capacités d'action de l'État culturel. Enfin, le secteur de l'économie du livre, il est vrai fortement impacté par les évolutions technologiques et la mondialisation économique, semble aujourd'hui faire l'objet de davantage d'attention de la part de l'État que le secteur, non marchand mais non dénué de poids économique, des bibliothèques.

On peut à l'inverse y trouver le signe d'une capacité de l'État à agir sur le long terme, qui manque dans bien des secteurs. La frénésie législative ou réglementaire n'est pas toujours un gage d'efficacité en politique. En matière de lecture publique, le rôle respectif de l'État et des différentes collectivités territoriales paraît relativement clair, bien plus en tout cas que dans le domaine du patrimoine, de l'éducation artistique ou du spectacle vivant, par

16. Philippe Poirrier, «La construction historique de l'État culturel», Politiques et pratiques de la culture, Paris, La Documentation française, 2010, p. 14. 
exemple. Le principe d'une compétence partagée est assez généralement admis. La demande d'État y reste forte chez beaucoup d'élus. Il n'y aurait donc pas lieu de faire évoluer radicalement un dispositif qui donne globalement satisfaction.

Il faut cependant évoquer ici la prise de position discordante de l'ABF, intervenue en 2007, à l'occasion du débat sur la réforme du dispositif de mise à disposition de conservateurs d'État dans les BM classées ${ }^{17}$. L’ABF, par l'intermédiaire de son bureau national, appela alors à " une décentralisation intégrale » en matière de bibliothèques. Celle-ci se caractériserait notamment par le transfert de la propriété des fonds d'État conservés dans les bibliothèques territoriales et la suppression, au nom de "l'orthodoxie administrative et budgétaire », des dispositifs dérogatoires (concours particulier et mise à disposition de conservateurs d'État), avec transfert des ressources correspondantes aux collectivités. Une loi sur les bibliothèques aurait défini la place respective des différentes collectivités (régions, départements, communes, intercommunalités). L'État n'aurait plus eu dans ce domaine qu'un rôle d'évaluation et de contrôle technique.

Cette analyse, qui ne semble plus faire partie de la doxa actuelle de l'ABF, est rappelée ici en tant que contribution au débat et non dans un but de stigmatisation. Elle est contestable sur plusieurs points :

$\odot$ La plupart des politiques culturelles sont fondées sur des dispositifs dérogatoires : la loi sur le prix unique du livre est dérogatoire, le statut des intermittents du spectacle est dérogatoire, la protection des monuments historiques est dérogatoire, le financement du cinéma est dérogatoire, les quotas de diffusion radiophonique de chansons françaises le sont également, etc. C'est précisément à l'existence de ce type de dispositifs que 
l'on reconnaît le volontarisme politique. C'est parce que le concours particulier est dérogatoire, qu'il est préservé dans son périmètre budgétaire actuel et que l'État continue de disposer de moyens d'action conséquents dans ce domaine. Un éventuel transfert de cette ressource aux collectivités, outre son caractère très hypothétique par les temps qui courent, la diluerait totalement et lui ferait perdre toute efficacité.

$\odot$ Si le maillage du territoire en bibliothèques est aujourd'hui globalement satisfaisant, il n'est pas encore totalement achevé, notamment dans les territoires qui connaissent un fort dynamisme démographique, comme les zones périurbaines ${ }^{18}$. De plus, un effort très important de requalification et d'adaptation aux nouveaux usages des bâtiments construits depuis une ou deux générations (comme la bibliothèque de la Part-Dieu à Lyon, pour ne citer qu'un seul exemple) sera à prévoir dans les prochaines années. Dans ce domaine, outre la poursuite de l'accompagnement financier de l'État, une réflexion collective, basée sur la mutualisation des expériences et pilotée nationalement, serait intéressante. Même si l'État n'a plus aujourd'hui le monopole de l'expertise dans le domaine des bibliothèques, ni celle de l'ingénierie, sa parole me semble conserver son utilité sur le plan technique, tant en direction des élus que des professionnels.

$\odot$ La crise des finances publiques commence à toucher sérieusement les bibliothèques et risque de durer. Les signes d'un retournement de tendance, après des décennies d'expansion, sont sous nos yeux. D’après les dernières statistiques du ministère de la Culture, l'investissement public en faveur des BM a baissé de $42 \%$ entre 2007 et 2012. Le nombre d'équivalents temps plein

18. Note de l'auteur, mars 2016 : voir, à ce sujet, le récent rapport de l'Inspection générale des bibliothèques (IGB) : L'Équipement des communes et groupements de communes en bibliothèques : lacunes et inégalités territoriales, décembre 2015. 
employés dans ces mêmes bibliothèques est également en recul sur la même période. Selon les derniers chiffres communiqués par la Sofia, qui portent sur l'année 2011, les achats de livres par les bibliothèques sont eux aussi en diminution. La mobilisation de tous les acteurs est indispensable pour amortir ce choc. En matière d'investissement, le principe des financements croisés, contrairement à une idée répandue, peut se révéler vertueux, s'il est mis en œuvre intelligemment, c'est-à-dire s'il s'appuie sur une analyse des territoires et de leurs besoins, en évitant le saupoudrage ou le simple guichet de distribution d'aides. Il contribue également à bonifier bien des projets et naturellement à corriger les inégalités territoriales.

$\odot$ La culture est par essence le domaine de la compétence partagée, de la coopération et du partenariat. Elle est l'affaire de tous et devrait le rester. Vouloir exclure l'État du jeu de la lecture publique serait un contresens. Vouloir par une loi, vieille revendication de l'ABF depuis sa création, fixer précisément les responsabilités des uns et des autres n'est pas forcément une bonne idée. L'absence de loi sur les bibliothèques n'a pas empêché l'essor de ces dernières décennies en matière de lecture publique. A contrario, il existe dans le domaine $\mathrm{du}$ tourisme plusieurs textes législatifs fixant la répartition des responsabilités entre les différents types de collectivités. C'est pourtant l'un des domaines où la clarification des compétences est la plus unanimement souhaitée. En matière de bibliothèques, le développement de la coopération interterritoriale, basée sur le volontarisme des acteurs et la liberté des initiatives locales, me semble préférable à la détermination d'un cadre rigide et uniforme.

La réforme territoriale qui s'annonce pose à nouveau la question du rapport entre l'État et les collectivités territoriales et celle de l'articulation entre les différents niveaux de collectivités. Cette 
réforme, si elle voit le jour, aura nécessairement des répercussions sur les bibliothèques, même si la culture n'est pas au centre des préoccupations dans ce dossier. Il est encore trop tôt pour en mesurer précisément les conséquences, tant l'impression qui se dégage pour l'heure est celle de l'improvisation et de l'incohérence $^{19}$. L'exécutif semble guidé par la devise du Marin Shadok: "Quand on ne sait pas où l'on va, il faut y aller... et le plus vite possible. » En outre, le spectacle de la réforme semble actuellement plus important que la réforme elle-même. Je me contenterai, faute de temps, d'évoquer ici quelques sources d'inquiétude et quelques motifs d'espoir.

Les premières lois de décentralisation étaient un acte de liberté, de responsabilisation et de confiance envers les territoires et les élus qui les représentent. À l'inverse, le sentiment dominant depuis plusieurs années est plutôt celui d'une recentralisation larvée. On en voit des signes par exemple dans la réduction de l'autonomie fiscale des collectivités, singulièrement des régions, que l'on prétend pourtant renforcer aujourd'hui, dans les allers et retours concernant la clause de compétence générale, dans le discours dominant sur l'épaisseur excessive du mille-feuille, le trop grand nombre d'élus locaux, les doublons supposés ou encore sur le niveau jugé excessif des dépenses des collectivités. En trente ans, le changement de ton est saisissant. Il n'est pas exagéré de parler aujourd'hui de crise de confiance entre l'État et les collectivités territoriales.

La réforme annoncée devrait, si l'on comprend bien, renforcer le couple intercommunalités/régions. Or, les bibliothèques sont actuellement gérées principalement à l'échelon communal et à l'échelon départemental, le seul du reste pour lequel existe une

\footnotetext{
19. Note de l'auteur, mars 2016 : ce texte a été rédigé en juin 2014. Depuis, malgré les avancées législatives, la mise en place des métropoles et le redécoupage des régions, la perplexité demeure.
} 
obligation légale. Leur environnement territorial, administratif et politique risque donc bien d'évoluer.

Les bibliothèques sont fondamentalement des équipements de proximité. C'est au niveau local que les choses se jouent pour l'essentiel en ce qui les concerne. Elles ont sans doute à gagner à inscrire leur action dans des territoires élargis, ceux des bassins de vie, correspondant au "pays réel ». Mais l'intercommunalité, si elle a fait ses preuves ici et là, ne va pas toujours de soi dans le domaine culturel, notamment quand il s'agit de transférer des équipements, ou des réseaux d'équipements existants. Aujourd'hui encore, une large majorité des bibliothèques territoriales reste sous gestion municipale, surtout en zone urbaine. De plus, le passage sous statut intercommunal est rarement une source d'économie budgétaire, car il s'accompagne généralement d'une montée en qualité des services rendus. Un transfert n'a de sens en effet que s'il entraîne une amélioration des prestations offertes à la population. Or, le coût de ces services n'est pas toujours proportionnel à la population desservie. L'effort à fournir est donc potentiellement conséquent. Pourra-t-il être assumé dans la durée ? De plus, en milieu rural, c'est-à-dire sur les deux tiers du territoire national, le maintien d'un échelon départemental, à même d'accompagner et de coordonner les initiatives locales en favorisant le travail en réseau, et de veiller aux nécessaires solidarités territoriales, ne serait probablement pas inutile.

Les attentes vis-à-vis des régions sont plus incertaines. C'est probablement à ce niveau que les initiatives prises sous l'ère Gattégno ont été le moins suivies d'effet. Les régions se sont peu investies dans la lecture publique, sans doute parce qu'elles ne gèrent pas directement des bibliothèques, parce que leurs moyens financiers sont limités, peut-être aussi parce que cet échelon, qualifié par certains d'« introuvable » dans le domaine des bibliothèques, correspond mal au cadre courant de leurs actions, même en 
matière de coopération. Les agences régionales pour le livre et la lecture, lorsqu'elles existent encore, sont au mieux des lieux d'échanges interprofessionnels et jouent rarement un rôle réellement opérationnel. Il n'est pas sûr que des régions redécoupées, aux territoires élargis ${ }^{20}$, correspondent mieux aux enjeux des bibliothèques.

Des raisons d'espérer demeurent cependant. La culture devrait, comme le sport, rester une compétence partagée ${ }^{21}$. Une autre avancée positive de ces derniers mois est la reconnaissance de la diversité des territoires, notamment dans la loi MAPTAM de décembre 2013. Le droit à l'expérimentation, la nécessité de trouver des formes d'organisation différentes selon les territoires, semblent aujourd'hui admis. Cela correspond assez, je le crois, aux besoins et à la nature des bibliothèques, qui ont fait preuve ces dernières décennies d'une grande plasticité. Elles ont su dans l'ensemble s'adapter à des changements considérables de leur environnement. Elles ont quelques atouts pour s'inscrire dans les évolutions territoriales en cours et expérimenter elles aussi de nouvelles formes d'organisation : elles ne sont pas en concurrence les unes avec les autres; pour elles, la coopération est une ardente nécessité, aucune n'étant autosuffisante. Elles ont intérêt à construire collectivement leur avenir.

L'État pour sa part, outre le maintien de sa politique de soutien des investissements des collectivités, devrait veiller en priorité à offrir aux bibliothèques un cadre juridique, économique et technologique adapté pour leur permettre de poursuivre leurs missions dans le nouvel environnement numérique qui se construit,

20. Note de l'auteur, mars 2016 : territoires considérablement élargis pour certaines d'entre elles, la nouvelle région Aquitaine - Limousin - Poitou-Charentes dispose ainsi d'un territoire aussi étendu que le Portugal ou l'Autriche !

21. Note de l'auteur, mars 2016 : la loi NOTRe du 7 août 2015 a supprimé la clause de compétence générale, rétablie précédemment par la loi MAPTAM du 27 janvier 2014, pour les départements et les régions. Toutefois, leurs compétences restent très larges et ils conservent la faculté d'agir notamment dans le domaine culturel. 
ce qui est loin d'être le cas aujourd'hui. Il devrait aussi œuvrer à renforcer, bien plus qu'aujourd'hui, la mise en réseau des bibliothèques et la mutualisation de leurs moyens, notamment en s'appuyant davantage sur ses opérateurs nationaux, $\mathrm{BnF}$ et bibliothèque publique d'information (BPI), dont l'attention aux territoires est encore insuffisante.

Ce qu'il manque aujourd'hui aux bibliothèques, c'est une vision d'ensemble portée par un discours commun. Sur ce point entre autres, un nouveau Jean Gattégno nous serait sans doute utile. 


\section{Peut-on, aujourd'hui encore, être fidèle à la pensée de Jean Gattégno?}

par Denis Llavori

Cette brève intervention a une ambition modeste : apporter un témoignage sur l'efficacité, la pertinence et même l'actualité des outils proposés par Jean Gattégno pour le développement des bibliothèques et de la lecture. Mon ambition n'est donc pas de proposer un bilan exhaustif de l'action de ce prestigieux directeur du livre, ce qui constituerait d'ailleurs une gageure (pour ne pas dire une folie) en quelques pages ! Elle est plutôt de rappeler des éléments de contexte pour susciter à l'issue un échange avec les auditeurs présents dans cette salle.

\section{Introduction}

Qu'il me soit permis, pour débuter, d'évoquer un souvenir personnel (et ancien : 1987 !) non pas parce que j'en suis le centre, bien évidemment, mais parce que Jean Gattégno, auquel nous rendons hommage sur ces deux journées, y occupe encore aujourd'hui toute la place. À ma sortie de l'Enssib me fut confiée la direction de la BCP du Cantal. Issu du concours externe, et ayant toujours vécu dans de grandes villes, je n'avais qu'une vague idée de ce que pouvait représenter la lecture publique en milieu rural, car mon expérience d'usager (la seule dont je disposais à l'époque) était bien éloignée de cette réalité. J'avais donc prudemment demandé à mon prédécesseur de bien vouloir me recevoir quelques jours avant ma prise de poste, pour un passage de relais. Ce qui fut fait, 
durant une journée, au mois d'août 1987. Et la conversation roula quasiment exclusivement autour d'un seul sujet : la circulaire Gattégno. Vous le savez, bien sûr, il s'agit de cette circulaire en date du $1^{\text {er }}$ août 1985 intitulée "Missions, moyens et fonctionnement des bibliothèques centrales de prêt ". Gattégno pour moi à l'époque, c'était donc d'abord et avant tout le nom d'une circulaire...

La BCP du Cantal avait été l'une des sept bibliothèques pilotes au niveau national pour le prêt en milieu scolaire : elle était très fortement engagée dans la desserte des écoles maternelles, primaires et même des collèges du département, au point de ne faire plus que cela. La circulaire Gattégno venait nous rappeler (après, il est vrai, la circulaire Groshens de 1978) que les BCP (je cite) «n'étaient plus dans de très nombreux cas que les auxiliaires de l'école primaire, et avaient failli à leur mission d'origine, à savoir la desserte de l'ensemble du public des petites communes ". Le style est direct et clair, et finalement peu habituel dans ce type de document, au ton généralement plus mesuré. Il résume sans doute ce que j'ai perçu de l'homme Gattégno à ce moment-là : franchise, conviction, intégrité intellectuelle et volonté sans faille.

Cette impression devait être confirmée cinq mois plus tard, le 29 janvier 1988, puisque l'ensemble des directeurs de BCP furent invités par Jean Gattégno à une réunion solennelle à la BN (qui n'était pas encore « de France »). Ce fut mon premier contact avec l'homme Gattégno, étant entendu que je n'étais qu'un des nombreux participants (le plus jeune, sans doute) et que j'écoutais consciencieusement sa parole, tassé dans mon fauteuil. Les BCP avaient été transférées aux conseils généraux des départements le $1^{\text {er }}$ janvier 1986, et les troupes avaient besoin d'être rassurées, notamment sur le rôle que l'État continuerait à jouer en faveur de la lecture publique. Et la fameuse 
circulaire ressurgit dans le débat, bien sûr. Aujourd'hui encore, en page 37 du rapport que l'IGB a récemment rendu (en novembre dernier) à madame la ministre de la Culture sur les devenirs des BDP, on trouve cette notation sous la plume de Jean-Luc Gautier-Gentès (je cite): "les BDP dans ces années-là se trouvent sous la double influence de leur récente décentralisation (1986) et de la circulaire dite “Gattégno”, rédigée un an avant dans cette perspective (1985). Des influences qui, dans une certaine mesure, s'exercent en sens contraire, la première (la décentralisation) poussant à la différenciation tandis que la seconde se voulait applicable à toutes ». Cela est évidemment exact mais les deux influences ne sont pas contradictoires. Sur ce sujet délicat, je fus donc rassuré par la parole de Jean Gattégno qui, avec autorité, mais aussi avec empathie et force de conviction, et surtout de manière très directe, nous confirma les trois points suivants :

1. la décentralisation, projet politique global, devait être saisie comme une chance pour la lecture publique, et non pas comme un risque ;

2. parallèlement, l'État n'abdiquait pas sa responsabilité dans la lecture publique, mais adapterait simplement ses modes d'intervention ;

3. le développement de la lecture publique en France était à ses yeux un projet majeur et global, qui devait impliquer tous les établissements de lecture publique, quelles que soient leurs autorités de tutelle et quelle que soit leur taille (BM classées, BCP, petites unités en milieu rural [les fameuses « petites unités de lecture publique (PULP) »], bibliothèques municipales de taille intermédiaire).

Ce discours, je dois le dire, me fit un bien fou : j'en suis sorti remotivé, disposant d'un axe de travail clair et rassuré sur la vigilante attention que nous portait l'État. 
Je souhaite revenir rapidement sur ces trois aspects qui, du point de vue de ma modeste expérience, synthétisent la doctrine de Jean Gattégno sur le sujet qui nous occupe dans cette table ronde. Comment d'ailleurs pourrais-je lui rendre un meilleur hommage que celui-là ? Ils ne sont pas si nombreux les hauts fonctionnaires qui peuvent, vingt-cinq ans après avoir quitté leurs fonctions, se targuer d'inspirer encore la pratique quotidienne de nombreux agents publics. Car il était - et il reste toujours sur de nombreux sujets - notre référence.

\section{Développements}

\section{$1^{\text {er }}$ point}

La décentralisation est une chance pour la lecture publique: c'était sa conviction profonde, il nous la faisait partager avec fougue. L'avenir lui a donné raison. Nul ne peut contester, je crois, qu'après plus trente ans de décentralisation des principaux équipements de lecture publique, le pari est très largement gagné. Les collectivités locales se sont approprié leurs équipements, et parfois jalousement. Au-delà de leurs fonctions traditionnelles, les BM des grandes communes sont également utilisées comme des outils d'aménagement ou de rééquilibrage des territoires urbains, voire comme marqueur d'image (fonctions également importantes que l'État n'éprouvait pas le besoin de leur imposer). Je pense - en leur temps - à Mériadeck à Bordeaux, ou à la Maison du livre, de l'image et du son de Villeurbanne. Je pense à des réalisations plus récentes : la Cité du livre à Aix-en-Provence ou l'Alcazar à Marseille. Les exemples pourraient être multipliés. On m'objectera qu'un symbole ne garantit ni une politique ni une action et que le fonctionnement de la structure est plus important que le geste architectural. Cette objection est fondée, bien sûr. Mais nous sommes dans un domaine - la culture - où la dimension symbolique 
reste très importante, et faire d'une bibliothèque l'emblème d'une politique municipale (ou départementale, songeons à la récente et superbe réalisation Pierresvives dans l'Hérault) ne peut que contribuer à la légitimité et à la popularité de ce type d'établissement. Et les efforts des collectivités territoriales pour leurs équipements de lecture publique, au niveau du fonctionnement cette foisci, sont tout aussi indéniables. Les rapports annuels de l'IGB, les bilans statistiques publiés par le Service du livre et la lecture (SLL) et la presse professionnelle (Bulletin des bibliothèques de France, Livres Hebdo ou la revue Bibliothèque(s) de l'ABF) en témoignent régulièrement. Il ne s'agit pas de dire que tout est parfait, mais les collectivités locales ont, depuis 1982, massivement construit des mètres carrés de bibliothèques, investi dans leur informatisation et leur équipement, recruté et formé des personnels et soutenu leurs établissements dans leurs efforts d'innovation et de modernisation, parfois même dans leurs plus audacieuses expérimentations (je pense par exemple à l'expérimentation que conduisent actuellement, au profit de tous, quelques grandes BM autour du projet Prêt numérique en bibliothèque [PNB]). De plus, cette proximité nouvelle de la tutelle a progressivement conduit les professionnels à mieux prendre en compte leurs publics, pour les placer aujourd'hui au cœur de leur réflexion et de leurs préoccupations. Jean Gattégno avait vu juste : la décentralisation a constitué une chance historique pour la lecture publique. Mais tout ceci n'aurait pas pu se faire dans d'aussi bonnes conditions sans l'accompagnement de l'État. Et nous en venons à la seconde promesse de Jean Gattégno :

\section{$2^{\mathrm{e}}$ point}

L'État n'abdiquera pas sa responsabilité vis-à-vis de la lecture publique. Dans un discours qu'il a prononcé au congrès annuel national de l'ABF en 1987, il synthétisait parfaitement le rôle de 
soutien et d'accompagnement qui était désormais celui de la DLL. Avec le ton direct qui était le sien, il déclarait alors : « la DLL a délibérément cessé de considérer les bibliothèques et les bibliothécaires comme étant en situation d'assistance perpétuelle ». Les collègues sous perfusion de l'époque ont certainement apprécié la formule... Puis il enchaînait en détaillant les moyens proposés pour permettre aux bibliothécaires d'assumer cette toute nouvelle autonomie. Je rappelle pour mémoire ces six propositions :

$\odot$ des outils techniques (base bibliographique, centre de coopération, etc.) ;

$\odot$ des relais locaux (structures régionales de coopération et associations travaillant au développement de la lecture) ;

๑ des réseaux destinés à réduire l’isolement des professionnels ;

$\odot$ le développement de la formation ;

$\odot$ la déconcentration de crédits d'intervention aux profits des conseillers régionaux pour le livre des directions régionales des affaires culturelles (DRAC) ;

$\odot$ et enfin la poursuite des opérations de construction de bibliothèques.

Force est de constater que ces six promesses ont également été tenues, et qu'elles sont aujourd'hui plus d'actualité que jamais. Sans entrer évidemment dans le détail, on peut rappeler pour deux d'entre elles - à titre d'exemple - cette actualité : les structures régionales de coopération que Jean Gattégno appelait de ses vœux existent aujourd'hui partout, et leur rôle est primordial, en matière de formation, de coordination, d'édition locale, de conservation partagée, etc. Et sur le sujet des opérations de construction, constatons aujourd'hui encore la pertinence et l'efficacité redoutable de l'outil spécifique imaginé par l'administration, le fameux « concours particulier », toujours opérationnel. Vous le 
savez, il s'agit de crédits inscrits au budget de l'État au titre de la construction et du fonctionnement des bibliothèques, qui ont été « fléchés » (en fait, sanctuarisés et préorientés) au sein de la dotation générale de décentralisation pour aider les collectivités locales à se doter d'un équipement de lecture publique. Cet outil permet à l'État d'imposer à ses partenaires des normes nationales qui garantissent l'accès de tous les Français à une même qualité d'équipement (ce qui est son rôle), quelles que soient les lignes de fracture (fracture sociale, fracture territoriale ou géographique, fracture numérique, pour ne citer que les plus évoquées d'entre elles). En contrepartie, les collectivités partenaires sont assurées d'un taux d'intervention élevé, et de la reconduction d'année en année des crédits ainsi affectés. Ce dispositif a été, au fil du temps, amendé, amélioré, mais globalement maintenu dans sa philosophie initiale. Nous sommes nombreux, dans la profession, à pouvoir témoigner de sa pertinence et de son efficacité. Le directeur de Médiathèque départementale que je suis n'aurait jamais pu, sans ce dispositif, inciter et accompagner les communes et communautés du département du Cantal à construire des établissements de lecture publique aux normes, largement ouverts au public, informatisés et gérés par des professionnels salariés. Il existe pourtant aujourd'hui dans le département 27 véritables médiathèques municipales ou intercommunales installées dans des communes de 6600 à moins de 1000 habitants (le Cantal est un département très rural, dont 259 communes sur 260 comptent moins de 10000 habitants). Un service de lecture publique de qualité et de proximité, impossible sans l'accompagnement de l'État. Là aussi, Jean Gattégno a tenu parole.

\section{$3^{e}$ point}

Le développement de la lecture publique est un projet global qui doit impliquer tous les acteurs. État et collectivités territoriales, 
bien sûr, nous venons de l'évoquer. Mais Jean Gattégno insistait sur la coopération des établissements de lecture publique : entre eux (catalogues collectifs, conservation partagée), avec d'autres établissements culturels publics (services d'Archives, centres de formation professionnelle, agences régionales du livre, voire musées ou écoles de musique), et avec tous les opérateurs privés de la lecture (libraires, auteurs et illustrateurs, éditeurs, relieurs, imprimeurs, conteurs, etc.). Dans le discours déjà évoqué qu'il a prononcé devant le congrès annuel national de l'ABF en 1987, il insiste : " la Direction du livre et de la lecture est engagée dans une politique de coopération, suivant en cela les recommandations du rapport rédigé par l'inspecteur général Louis Yvert. Ce rapport est l'un des éléments importants de la réflexion menée par l'administration dans le cadre des lois de décentralisation ". Et de poursuivre en citant (et en commentant) : "le centre de coopération des bibliothèques de Massy, l'intervention financière pour le fonctionnement des associations régionales de coopération, la promotion du travail en réseau régional coordonné à l'échelle nationale pour l'informatisation, ou encore la mise au point de normalisations en matière de bibliographie informatisée, de logiciel de prêt ou de statistiques ». Si la terminologie utilisée accuse parfois son âge (les termes de bibliographie informatisée et logiciel de prêt ne sont plus guère employés), la philosophie, l'inspiration de cette action existent plus que jamais. Quel établissement de lecture publique - quelle que soit sa taille - peut aujourd'hui prétendre agir efficacement en dehors de tout réseau et sans coopérer? Ici également, Jean Gattégno a tenu parole. Il a même été, sur ce dernier sujet, visionnaire (puisqu'à l'époque les réseaux numériques n'étaient pas ce qu'ils sont devenus) : car l'avenir (pour ne pas dire la survie) de la lecture publique passe incontestablement aujourd'hui par la coopération et le fonctionnement en réseau. J'en veux pour preuve la volonté 
récemment exprimée par madame la ministre de la Culture, dans son discours prononcé le 24 mars dernier en ouverture du Salon du livre de Paris : " concernant la bibliothèque publique d'information, sa future directrice [...] aura pour mission de donner une nouvelle impulsion [...], et j’y tiens car il s'agit d'une des raisons profondes de l'existence de cet établissement, pour réactiver son rôle d'animation et de lieu de ressources au service du réseau de la lecture publique ». La mission " coopération » de la BPI est ainsi clairement identifiée comme stratégique.

\section{Conclusion}

Et aujourd'hui ? L'annonce d'une réforme de l'organisation territoriale française va avoir des conséquences sur les politiques publiques de la lecture de nos territoires. Sans entrer dans le détail d'un projet qui - s'il est annoncé - n'est pas encore adopté, nous pouvons néanmoins avancer quelques réflexions. La mise en œuvre de la lecture publique a, depuis les lois de décentralisation de 1982, été l'affaire des communes et des départements, presque exclusivement. Puis on a vu s'amorcer un mouvement de fonds régulier de mutualisation des équipements municipaux dans le cadre de l'intercommunalité. C'est ainsi le cas lorsqu'une métropole, une communauté d'agglomération ou une communauté de communes (bref, un établissement public de coopération intercommunale) prend - comme la loi l'y autorise - la compétence " lecture publique » ou " grands équipements ». Mais si les départements disparaissent, et qu'une partie de leurs compétences est transférée aux intercommunalités, la lecture publique sera alors partout une affaire communautaire. Les périodes de changement sont nécessaires et l'évolution, l'adaptation sont vitales pour la lecture publique. Mais ces périodes d'incertitude sont également anxiogènes 
pour les personnels affectés dans les établissements. Et l'on ressent aujourd'hui cruellement l'absence à nos côtés de Jean Gattégno : qu'en aurait-il pensé ? Qu'aurait-il dit pour nous rassurer et nous accompagner? Mais il faut désormais que nous fassions sans lui... 


\section{Trente-trois ans après sa publication, relire «Du pécheur au militant »}

par Benoît Tuleu

Je veux parler ici d'un sujet qui, s'il relève de l'intime, n'était pour Jean ni secret, ni insignifiant, ni fondamental: son homosexualité. Pour éviter l'écueil d'une approche essentialiste ou trop personnel, mon intervention s'en tiendra à ce que Jean lui-même a pu écrire sur le sujet : je remercie donc Marc Olivier Baruch et Gérald Grunberg d'avoir remis sous mes yeux l'article que Jean avait publié en 1981 dans la revue Le Débat, intitulé « Du pécheur au militant ${ }^{22}$ ».

Il s'agit d'une synthèse sur la question homosexuelle en Occident, en particulier en France, Grande-Bretagne, Allemagne et États-Unis depuis la période moderne et surtout depuis le XIX siècle. Construit, référencé, plein d'intelligence, ce texte convoque l'histoire, la littérature, la politique, les sciences humaines et sociales, dans une forme universitaire classique mais avec un socle d'engagement militant qui, lui, ne l'est pas : la question du droit fondamental à la liberté des hommes et femmes homosexuels, en effet, n'y est pas défendue, mais elle est présente à chaque ligne comme une affaire entendue d'avance avec le lecteur, entre gens intelligents et de bonne compagnie. Le titre résume l'ensemble du propos de Jean Gattégno : la figure de l'homosexuel, homme ou femme, a évolué dans cette période de celle du pécheur que l'on punit à celle du militant qui revendique ses droits.

Alors, trente-trois ans plus tard, dans la France du mariage pour tous péniblement mais finalement légalisé et de l'homoparentalité

22. Jean Gattégno, «Du pécheur au militant », Le Débat, 1981/3, n 10, pp. 118-131. [En ligne] : < www.cairn.info >. 
qui commence à avoir droit de cité, quelle lecture peut-on faire de ce texte?

Ce qui frappe à sa lecture, disons-le tout net, c'est sa pertinence et son actualité. Mais commençons par quelques éléments de contexte. L'article de Jean Gattégno est publié en mars 1981. La France pour encore quelques semaines est présidée par Valéry Giscard d'Estaing ; elle s'apprête à élire François Mitterrand mais pour l'instant et depuis vingt-six ans, la droite est au pouvoir. L'article 331-3 introduit par Pétain en 1942 dans le Code civil sur proposition de Darlan, et conservé tel quel en 1945 par la Quatrième République, punit d'un emprisonnement de six mois à trois ans et d'une amende de 60 à 15000 francs quiconque aura " commis un acte impudique ou contre nature avec un individu de son sexe mineur de moins de vingt et un ans " (la majorité sexuelle pour les hétérosexuels étant à 15 ans). Le " délit d'homosexualité » légitime ainsi jusqu'en 1981 les descentes de police dans les bars et lieux de rencontre fréquentés par les homosexuels, les enfermant de facto dans la clandestinité. La violence, physique et verbale, à l'encontre des hommes et femmes homosexuels est courante et rien ne l'interdit particulièrement, au contraire : par une loi de juillet 1960, l'homosexualité est classée parmi les « fléaux sociaux » et le gouvernement est autorisé à « prendre toute mesure pour réprimer le délit d'homosexualité ».

C'est dire le courage qu'il faut pour signer de son nom un article aussi engagé, dans ce dossier intitulé «Histoire de l'homosexualité » au sommaire de la toute jeune revue Le Débat, en ce printemps $1981^{23}$.

\footnotetext{
23. Notons au passage que si sur cette question le courage de Jean Gattégno et de Michael Pollak est resté intact comme le prouvent leurs parcours respectifs, il n'en va pas de même pour la ligne éditoriale de la revue Le Débat devenue depuis une tribune pour les pourfendeurs de la légalisation du mariage des couples de même sexe et de l'homoparentalité, habillés du vernis d'« experts » en psychanalyse ou en anthropologie. D'ailleurs la revue, bien mal nommée, avait entamé très tôt son virage néoconservateur ; dès 1990 elle était par exemple porte-drapeau des opposants les plus caricaturaux au projet d'une nouvelle Bibliothèque de France, future BnF.
} 
L'article de Jean Gattégno est ainsi entouré d'un texte de l'historien archéologue Alain Schnapp sur «Une autre idée de l'homosexualité en Grèce antique » et une étude du sociologue américain Michael Pollak sur «Les vertus de la banalité », synthèse de deux recherches sur la condition des gays à la fin des années 1970. La fin du dossier est consacrée à un compte rendu par Jean-Claude Schmitt de l'article de John Boswell «Christianisme et homosexualité au Moyen Âge » publié l'année précédente aux États-Unis.

Il est significatif que Jean Gattégno, brillant universitaire, spécialiste de Lewis Carroll et d'Oscar Wilde, ait pris part à cette publication qui fera date pour les militants de l'émancipation homosexuelle, dans un contexte où leur matériel théorique est rare. L'engagement de ces intellectuels (chercheurs, universitaires), pas nécessairement homosexuels d'ailleurs, relaie en effet un important mouvement associatif et militant, lui-même heureusement "récupéré » par les partis de gauche qui vont arriver au pouvoir. Le terrain militant est occupé par le très radical Front homosexuel d'action révolutionnaire (FHAR), et son pendant réformiste, le Comité national des homosexuels (CNH) ainsi que les Comités unis anti-répression des homosexuels (CUARH), dont les revendications sont soutenues par la Ligue des droits de l'homme. Ils constituent un maillage fragile mais présent et actif dans toutes les villes du pays. L'arsenal répressif reste entier mais ce réseau militant est soutenu par des intellectuels, des associations progressistes et des partis politiques.

Sur les éléments de l'article eux-mêmes, mentionnons maintenant plusieurs points remarquables qui le rendent contemporain et toujours pertinent.

๑ La description de la répression de l'homosexualité par la médecine, du XIX siècle à nos jours, au moyen de dispositifs atroces justifiés par les impératifs de santé publique. Rappelons que ce n'est qu'en 1992 que l'Organisation mondiale de la santé 
raye l'homosexualité de la liste des maladies mentales. Dans la prise en charge des "déviants » ou "pervers », le relais est également pris par une certaine psychanalyse normative qui s'abrite derrière les prises de position de Wilhelm Reich et de Jacques Lacan, qui passaient - et étrangement passent encore - pour des libérateurs.

$\odot$ La connaissance experte de la situation de la Grande-Bretagne, que ce soit sur le plan de la législation, de la morale sexuelle victorienne, de l'obsession du scandale dans la haute société anglaise... Ce miroir tendu par le spécialiste français d'Oscar Wilde et de Lewis Carroll permet de comprendre les rouages de ce qui est aujourd'hui en œuvre dans les débats sur le coming out et le outing. Il met en lumière l'incompréhensible et persistante réticence d'une partie des élites sur les questions d'énonciation et d'engagement personnel - questions que Jean Gattégno, il y a trente-trois ans, réglait avec élégance, notamment en signant cet article.

$\odot$ Au-delà de celle de la droite, cas désespéré, la dénonciation d'une certaine gauche très réservée, voire franchement hostile, sur la question de l'émancipation homosexuelle : le texte pointe par exemple les prises de position staliniennes de membres importants de la direction du Parti communiste (les Vermeersch, mais aussi Jacques Duclos, s'appuyant sur des écrits de Engels), mais aussi la frilosité des "socialistes » anglais, soucieux de préserver la famille ouvrière traditionnelle.

$\odot$ Le souci de traiter le sujet de façon globale, non seulement de façon interdisciplinaire mais sur une aire géographique étendue ; et, dans le même esprit, de réfléchir à l'ensemble du genre humain concerné, en intégrant à la réflexion la situation des lesbiennes qui est souvent le point aveugle des études sur l'homosexualité, en particulier quand elles sont conduites par 
des hommes. Jean Gattégno fait ici preuve d'un universalisme concret et qui apparaît très pertinent à l'heure de la convergence des luttes «lesbiennes, gays, bi, transgenres et intersexuels (LGBTI)».

Enfin, on peut relever avec jubilation que l'élégance et la précision du propos n'excluent pas l'humour, y compris quand il s'agit de céder à un jeu de mots au goût discutable ; ainsi, à propos des provocations des policiers américains dont la mission est de piéger les homosexuels dans leurs lieux de rencontres en se faisant passer eux-mêmes pour des gays, le texte expose que le but est « de les prendre, si j’ose dire, la main dans le sac »... En forçant à peine le trait, on pourrait dire que ce type d'humour fait partie de l'affirmation nouvelle de la condition homosexuelle. Peut-être est-il utile de rappeler ici que le mot " gay » était en 1981 en train de s'implanter en France et que son usage consiste à affirmer la gaieté dans tous les sens du terme : il s'agit d'être heureux et joyeux malgré et contre la sinistre et violente répression, et aussi malgré et contre sa version compassionnelle, plus soft, incarnée alors par Ménie Grégoire, auteure dix ans plus tôt d'une émission de radio intitulée « L'homosexualité, ce douloureux problème » (10 mars 1971).

Plus sérieusement, l'article se termine par une réflexion sévère et malheureusement visionnaire. D’abord, Jean Gattégno constate la maladresse et la fragilité d'un front associatif militant qui en 1981 est animé par une radicalité de façade et en réalité une impuissance à se structurer en véritable mouvement. Il critique la résignation collective des homosexuels à subir les tracasseries, le harcèlement et le mépris qui sont le revers d'une tolérance a minima, sans droits réels ; cet homme épris d'engagement collectif fustige « leur stratégie individuelle permettant à beaucoup, en fait, toujours aux mêmes, de passer sans dommage au travers des obstacles ». Magistrale démonstration de conscience politique où l'on devine que ces questions abstraites sont incarnées 
dans des situations qu'il a quotidiennement vécues et que vivent encore bien des homosexuels.

Enfin Jean Gattégno conclut son étude sur l'inquiétude suivante : « En France, à l'automne 1980, les deux chambres du Parlement ont refusé d'abroger la discrimination antihomosexuelle introduite dans la loi par Pétain en 1942, le tout sans que la gauche traditionnelle s'émeuve outre mesure. Entrons-nous dans une nouvelle phase d'ordre moral ?»

Deux mois plus tard, cette même gauche arrive au pouvoir. Elle abrogera le délit d'homosexualité le 4 août 1982 (dès juin 1981, une circulaire du nouveau ministre de l'Intérieur Gaston Defferre demande aux forces de police de cesser les interventions dans les lieux de rencontres homosexuels).

Mais le 8 juin 1981, un mois encore plus tard, un article de la revue du Center Desease Control (CDC) d'Atlanta mentionne pour la première fois les maladies multiples dont meurent de façon inexplicable et foudroyante de jeunes hommes homosexuels aux États-Unis. À l'automne 1981, à Paris, le jeune Dr Willy Rozenbaum fait le lien entre cet article et les cas de malades auxquels lui-même est confronté.

L'épidémie de sida a commencé. Bien sûr, elle s'étendra bien audelà de la population homosexuelle, mais elle favorisera la propagation d'un discours de haine homophobe et plus largement d'ordre moral, répondant finalement par l'affirmative à la question inquiète de la fin de l'article de Jean Gattégno. Il y aurait beaucoup à dire sur la finesse de son analyse de la situation de 1981 : individualisme et faiblesse de l'engagement collectif, mollesse de la gauche sur la question des droits, retour possible de l'ordre moral, aggravation des discours et procédés discriminatoires devant la situation de fragilité de la population homosexuelle encore majoritairement réduite à la clandestinité. 
Il est difficile de dire si le parcours, du pécheur au militant, dessine aussi sur cette question intime une sorte d'autoportrait de Jean Gattégno. Montrer l'évolution de l'un à l'autre, c'est de toute façon tracer le chemin de bien des personnes homosexuelles, audelà de sa génération. Sans doute lui-même avec sa subtilité toujours en éveil aurait-il aimé l'idée, développée dix ans plus tard par Eve Kosofsky Sedgwick dans Epistemology of the Closet ${ }^{24}$, que l'on ne sort jamais vraiment une fois pour toutes du placard, que le coming out et le chemin de l'émancipation sont à refaire presque quotidiennement, tant sont intériorisées les entraves de la condition minoritaire.

Avec le recul des trente-trois ans qui se sont écoulés, on peut d'ailleurs regretter que ce travail de chercheur universitaire, précurseur et engagé, n'ait pas été poursuivi par l'émergence en France d'études et de recherches sur la question homosexuelle. C'est aux États-Unis qu'ont émergé les Gay and Lesbian Studies et les Gender Studies, qui ont d'ailleurs emprunté à des penseurs français (Foucault, Deleuze, Derrida) leurs outils théoriques. En France au contraire, la promesse qui affleure dans l'intelligence pluridisciplinaire de cet article n'a pas été tenue. Hors l'exception que constitue le colloque organisé par Didier Eribon en juin 1997 au Centre Pompidou ${ }^{25}$, le développement des études et recherches sur la question homosexuelle n'a rencontré que de l'hostilité, au nom d'un catéchisme conservateur et cocardier qui invoque l'universalisme pour mieux exercer sa domination. La dernière sinistre illustration de ce processus de blocage a été la caricature de ce champ d'étude en prétendue "théorie du genre ", d'abord dans les milieux les plus homophobes et relayée ensuite un peu

\footnotetext{
24. Eve Kosofsky Sedgwick, Epistemology of the Closet, Berkeley, University of California Press, 1991. La traduction française, de Maxime Cervulle, a été publiée en 2008 par les éditions Amsterdam.

25. Lors de ce colloque sont notamment intervenus Leo Bersani, George Chauncey, Eve Kosofsky Sedgwick, Pierre Bourdieu, Monique Wittig. Les actes ont été publiés en 1998 par les éditions du Centre Pompidou.
} 
partout sans que les universitaires, à de rares exceptions près, ne se mobilisent contre une telle bêtise qui pourtant les menace tous, quels que soient leurs centres d'intérêt.

Il y aurait pourtant un grand bénéfice à réfléchir sur la démarche de Jean Gattégno écrivant cet article, sur cette manière particulière de s'engager en tant qu'intellectuel et chercheur, avec des outils universitaires mais en franchissant les frontières entre le monde savant et le monde profane, entre les différentes disciplines, mais aussi entre l'intime et le social, entre l'expérience de celles et ceux qui sont homosexuels et l'intérêt solidaire de ceux qui ne le sont pas. Sa démarche procède d'un heureux et généreux amalgame qui vise, sur ce sujet comme sur tous ceux qu'il a abordés, à l'émancipation individuelle et collective. Surtout, l'effacement de Jean Gattégno derrière son sujet n'empêche pas en le relisant d'y distinguer nettement son sourire, plein d'intelligence amusée et de bienveillance. 


\section{Jean Gattégno et la foi chrétienne, dialogue entre Véronique Chatenay- Dolto et François Suard}

\section{L'origine d'une vocation, le sens d'un engagement}

- Véronique Chatenay-Dolto

Jean Gattégno n'est pas né catholique, il a choisi le baptême. Nous qui avons connu Jean Gattégno plus tard dans sa vie, bien après ses années de formation, nous le savions croyant, catholique. Comment le savions-nous ? Pas par lui, qui se livrait peu à des confidences, veillant à ne pas mélanger les différents ordres de sa vie. C'est du moins ma perception. À la fin de sa vie, quand la maladie a pris le dessus, il nous a laissés l'approcher plus intimement. Pourtant je n'ai jamais osé ou voulu l'interroger sur sa foi. C'est après sa mort, pour préparer la cérémonie de SaintMerry, que nous avons rencontré les témoins de cette histoire, Antoine Delzant et vous, François Suard.

Le choix libre de la foi catholique est pourtant, je crois, le secret et la clé ou une des clés de cette personnalité si riche, et à mes yeux une dimension qu'il nous laisse en partage.

Peut-on (doit-on) expliquer ou comprendre ce qui a conduit un jeune intellectuel, un étudiant épris de liberté, à s'engager dans la foi catholique, à la fin des années 1950 ?

Peut-on expliquer le chemin qui l'a mené (il y a près de soixante ans) vers la demande du baptême, et l'inscription dans la pratique individuelle et collective du dogme catholique? 
François Suard, vous êtes l'un des premiers et des plus proches témoins de ce chemin spirituel, qui est aussi un chemin de l'amitié. Comment comprenez-vous le chemin vers le baptême dans la formation intellectuelle de Jean Gattégno?

Sans trahir l'intime d'une vie et des choix, votre témoignage nous permettra d'approcher la dimension spirituelle d'un homme et de son action, dimension qui à mes yeux est inséparable des autres engagements de sa vie.

Peut-on relier ou séparer formations spirituelle et intellectuelle? Comment l'engagement de Jean dans le syndicalisme, dans la vie associative, dans le souci de transmission vers les jeunes (en France et en Tunisie) est-il lié à son choix des valeurs chrétiennes?

La foi est-elle un pas de plus ? Vers où?

\section{- François Suard}

L'adhésion de Jean à la foi chrétienne est certainement le résultat d'un long cheminement, dont nous avons seulement été les témoins, n'en recueillant que l'aboutissement.

Jean venait d'une famille juive non religieuse : on ne peut donc parler de conversion, d'autant que la question de l'adhésion à une foi quelconque n'avait pour lui rien d'une urgence. Et le catholicisme des années 1950 était, au moins dans son expression institutionnelle publique, médiocrement attirant : c'était la fin du règne du pape Pie XII (il est mort en 1958), un règne jusqu'au bout autoritaire, marqué notamment par la crise des prêtres ouvriers ; une ouverture avait pourtant eu lieu dans le domaine liturgique, avec l'importance retrouvée de la liturgie pascale, rapprochée du public. C'était en même temps, mais hors de la tutelle ecclésiastique, une période de frémissement, et même de bouillonnement 
intellectuel, avec des institutions vivantes animées par des laïcs, comme le Cercle des intellectuels catholiques, où s'exprimaient des gens comme André Mandouze, Irénée Marrou, René Rémond, et où la trace de Mounier était encore vivante. Bouillonnement aussi parmi les théologiens, crossés par le pouvoir ecclésiastique, mais dont on se passait les bonnes feuilles ou les propos : Congar, De Lubac.

De tout cela, Jean pouvait avoir des échos. Il avait eu en khâgne des amis catholiques, avec lesquels il avait noué des liens étroits ; il les retrouvera à Ulm et continuera de les fréquenter jusqu'à la fin de sa vie. À l'École pourtant, malgré ces liens, d'abord intellectuels et culturels, malgré ses curiosités multiples - à quoi ne s'intéressait-il pas ? -, on pouvait recevoir de lui des leçons de cinéma, de science-fiction, de musique et de danses folkloriques, d'opéra - il apparaissait en recherche d'autre chose, on dirait aujourd'hui en recherche de sens. Il était à la fois entouré d'amis, et solitaire.

La rencontre du groupe tala d'Ulm a sans doute joué un rôle de déclic. C'était un groupe dynamique, aidé dans sa réflexion par un aumônier d'une grande qualité intellectuelle et humaine, André Brien, et formé de gens très différents les uns des autres, parmi lesquels se dégageaient de fortes personnalités comme Jean Rousselet chez les littéraires ou Antoine Delzant chez les scientifiques. Le groupe n'intervenait pas en tant que tel dans la vie de l'École, mais ses membres étaient connus et même reconnus. On n'était plus au temps où l'Union des étudiants communistes prétendait au monopole de la pensée et de l'action (Budapest, en 1956, était passé par là), et pas encore à celui du tout-révolutionnaire. Talas et non-talas se retrouvaient souvent dans la lutte contre le "national-molletisme », contre la guerre d'Algérie. Pour sa part, Jean était mendésiste, puis gaulliste, avant de se retrouver plus tard à l'aise dans le renouveau de la gauche. 
Ce que le groupe tala, et particulièrement certains de ses membres, dont j'étais, lui ont apporté, c'est probablement une famille spirituelle, dont il a découvert qu'elle s'accordait dans ses grandes lignes avec l'attente et les exigences qui étaient les siennes: mener une vie fraternelle, dont il n'avait peutêtre pas fait l'épreuve jusque-là, avec le souci de transmettre à d'autres les biens reçus. J'avais noué avec lui, dès notre arrivée à l'École, à la rentrée 1956, de solides liens d'amitié, et j'ai sans doute été le témoin privilégié de cette évolution paisible qui l'a conduit à se tourner vers le baptême. Je retrouve dans une lettre d'octobre 1958 les termes dans lesquels il m'annonce sa décision : " La vraie nouvelle, mais le mot est impropre, c'est que je suis allé voir Brien aujourd'hui pour lui dire que je désirerais recevoir le baptême. Te dire comment ça m'est venu, je ne le pourrai pas plus qu'à lui. Suite d'un long mûrissement sans crise, sans mysticisme, sans ferveur peut-être. Mais c'est un désir (une volonté) assuré... » Sans doute a-t-il trouvé aussi par la suite dans certains lieux de prière - je pense aux monastères de la Pierre-qui-Vire et du Bec-Hellouin - un bonheur à la fois esthétique et spirituel. Mais ce qui a vraiment compté pour lui dans la découverte du christianisme, c'est, je crois, Jésus et l'Évangile, avec la grande intuition chrétienne selon laquelle on ne peut approcher Dieu qu'à travers l'homme et ce qu'on fait avec lui et pour lui. Toutes les activités hors École qu'il avait depuis longtemps - le temps considérable qu'il consacrait à l'Union française des colonies de vacances par exemple - allaient dans ce sens. Sa vie, avec ses exigences, s'en est trouvée unifiée, apaisée, confortée : il reconnaissait comme son bien propre une part de mystère à lui destinée - le mystère divin - qu'il pouvait en même temps partager avec d'autres. Je ne pense pas qu'il allait chercher plus loin, pas plus que nous du reste. On récitait le Credo sans se poser de questions : pour nous, ce n'était pas l'essentiel, mais 
plutôt de savoir comment on pouvait, dans une action concertée grâce aux associations diverses dont nous faisions partie, mettre l'Évangile en pratique, comment on pouvait « chercher le royaume de Dieu et sa justice ", c'est-à-dire contribuer à construire un monde fraternel : on sait dans quels domaines Jean a œuvré, l'action populaire, l'activité syndicale puis le domaine immense de la lecture dans lequel il voyait un outil privilégié pour transmettre ce qu'il avait reçu. Il n'y avait donc pas, et il ne pouvait pas y avoir, d'opposition entre la foi qu'il avait découverte et les actions multiples qu'il a menées au cours de sa vie professionnelle. Du reste, il n'avait rien d'un prédicant, étant par nature avare de confidences : la foi était pour lui de l'ordre de l'intime.

\section{L’héritage de Jean Gattégno,} le chemin d'un homme parmi les hommes de bonne volonté

\section{- Véronique Chatenay-Dolto}

Syndicat général de l'Éducation nationale (SGEN), action dans les prisons, éducation populaire, les engagements politiques et sociaux de Jean Gattégno le situent du côté de ceux qui sont «à la gauche du Christ ». Si Jean Gattégno n'était pas un prosélyte, il était pourtant habité par un goût, une volonté de transmettre.

Chacun sait ce qu'il lui a transmis, des valeurs humanistes et fraternelles, ou un autre message, de nature plus spirituelle.

Face au double mouvement de sécularisation de la société et de raidissement de l'Église sur les questions de morale, je me demande quelles sont les interrogations, les convictions que nous pourrions formuler dans la fidélité de l'histoire de Jean. 
Notre époque est marquée par la sécularisation du message chrétien, par la dissémination, voire la dissolution des valeurs de charité et de fraternité : comment les intellectuels chrétiens aujourd'hui se situent-ils dans leur relation au message évangélique?

Peut-on réfléchir, dans la fidélité à Jean, à ce que signifie aujourd'hui l'écoute de l'Évangile ? Comment les chrétiens, dans un monde désenchanté, poursuivent-ils la recherche d'un message d'espérance?

\section{- François Suard}

La profonde mais discrète intrication de la foi chrétienne et de l'action publique, caractéristique de la vie de Jean, pourrait-elle exister encore aujourd'hui, étant donné les multiples changements qui se sont produits dans les mentalités et dans la place du christianisme en Occident, particulièrement en France ?

Si l'on résume à grands traits les cinquante dernières années, on pourrait dire que se sont succédé dans l'Église catholique une période de grande ouverture et de fécondité (les années 1960-1970), aboutissement là encore d'un long travail préparatoire, suivie d'une période - dans laquelle nous nous trouvons aujourd'hui - de rétraction et en même temps de dilution caractérisée par la perte progressive d'influence du catholicisme comme cadre de pensée et de vie collective.

Temps d'ouverture : c'est la période conciliaire (1962-1965), marquée par la réconciliation avec le judaïsme, l'affirmation de la liberté de conscience, la nécessité du dialogue entre les confessions chrétiennes, avec la parole rendue aux théologiens jusquelà réduits au silence, suivie du jaillissement de petites communautés rayonnantes, comme à Boquen en Bretagne, à la SainteBaume dans le Midi. 
C'est aussi le foisonnement d'initiatives et de pratiques auxquelles donne lieu l'ébranlement culturel de 1968, à l'écart duquel le catholicisme ne peut rester extérieur : multiplication des communautés, ébranlement de la conception hiérarchique de l'Église, départ de nombreux prêtres ou religieux mais aussi engagement clairement assumé d'un certain nombre de catholiques dans le renouveau de la gauche (à l'École, Jean reprochait parfois aux catholiques de ne pas s'engager politiquement, comme s'ils avaient peur de se compromettre).

Dès lors s'amorce pourtant dans l'institution un mouvement de repli, avec un magistère soucieux de conserver son autorité et d'affirmer l'orthodoxie : certains des théologiens en pointe avant et pendant le concile commencent à se crisper sur des positions doctrinales, comme Ratzinger, futur Benoît XVI, De Lubac ou Daniélou.

À l'inverse, dans la pensée des laïcs, l'encyclique de Paul VI Humanae Vitae, qui proscrit la contraception, fait figure de retour en arrière inacceptable et mine pour l'avenir le crédit que l'on peut accorder à tout message émanant du pape. L'arrivée de Jean-Paul II, en 1978, un pape non italien, redonne espoir, avec la liberté qu'il prend vis-à-vis du bloc de l'Est, avec ses initiatives en faveur des droits de l'homme ou de l'œcuménisme; mais lui aussi va défendre très vite l'autorité de l'institution, en bloquant l'essor de la Théologie de la libération en Amérique du Sud, en renouvelant les interdits sexuels (condamnation de la contraception, de l'avortement), en faisant de questions de coutume et de discipline (mariage des prêtres, ordination des femmes) des fondamentaux théologiques. Quant à son successeur, Benoît XVI, le souci de réintégrer les fondamentalistes dans le giron ecclésial semble avoir occupé une part essentielle de son activité, le départ silencieux de nombreux chrétiens progressistes ne lui posant apparemment pas de problème. En revanche, 
son abdication a réellement ouvert une porte dans la forteresse, en désacralisant la fonction du pape, qui redevient ce qu'il aurait toujours dû être, un serviteur qui renonce à sa charge quand il n'est plus en mesure de l'assurer.

Résultat aujourd'hui ? Crispation d'un côté, à la fois dans l'institution et chez certains catholiques, jeunes ou vieux, sur une conception de l'Église figée dans une attitude de contre-culture, distribuant des vérités intangibles aussi bien dans le domaine de la foi et des rites (messe en latin) que dans celui de l'organisation sociale, prônant une visibilité de la croyance (retour aux processions, à la sonnerie de l'angélus) : ce sont toutes les variantes soft ou hard du traditionalisme et de l'intégrisme qu'on a pu voir se déployer par exemple lors du débat sur le mariage pour tous.

De l'autre, étiolement progressif de la pratique religieuse, nonrenouvellement des clercs, et départ des catholiques les plus ouverts, ceux que Jean-Louis Schlegel appelle «la gauche du Christ ». Et parmi ces partants, en général discrets, il faut mentionner tout particulièrement les femmes, artisans premiers de la transmission de la foi, qui ne se reconnaissent plus dans une institution exclusivement masculine et dans une lecture des textes fondateurs ne relativisant pas comme marqueur d'une société patriarcale dépassée une conception de Dieu anthropomorphe ou plutôt andromorphe.

Sans doute existe-t-il entre ces deux types de position des catholiques nombreux, jeunes ou vieux, qui restent à l'aise dans l'institution et dans une formulation de la foi relativement traditionnelle, tout en étant attentifs aux autres : on les retrouve dans des paroisses ouvertes sur l'extérieur, avec des aspects charismatiques, dans certains mouvements comme l'Action catholique ouvrière, dans la récente Conférence des baptisés ; je rattacherai aussi à cette mouvance l'association Confrontations, héritière du 
Centre des intellectuels catholiques, animée essentiellement par des universitaires.

Sans préjuger de l'évolution future de ces groupes, on peut dire que leur vitalité, au moins pour l'instant, ne ralentit pas l'effacement progressif du catholicisme, qui n'est pas uniquement dû au rejet de l'institution et de certaines pratiques rétrogrades. C'est aussi le résultat d'une réflexion sur les objets de croyance et leur rapport à la foi. Les chrétiens dissidents dont nous parlons - ils ont maintenant plus de 70 ans - ont lu la Bible ; certains ont fait un peu de théologie, se sont interrogés sur les circonstances de composition des Écritures, sur les rapports entre les formulations qu'on y trouve et un contexte historico-sociologique donné ; ils ont suivi les découvertes scientifiques sur l'expansion de l'univers, sur l'évolution : le résultat est que le Credo catholique, censé exprimer la foi de l'Église, n'a plus de sens pour eux, si ce n'est de constituer un lien avec une tradition dont ils se reconnaissent les héritiers. Participe également de cet éloignement la défiance à l'égard de toute religion conçue comme un système de pensée et comme une marque identitaire, dont les dérives extrêmes (massacres interreligieux en Centrafrique, délires djihadistes en Syrie ou en Irak) renvoient aux erreurs tragiques du passé (les croisades, l'Inquisition, les guerres de Religion en Europe).

Ces partants, qui ne fréquentent plus les paroisses ou ne les retrouvent que de façon très épisodique, dont les petits-enfants ne sont pas baptisés parce que leurs enfants se sont eux-mêmes écartés de la foi, se regroupent dans de petites communautés de réflexion spirituelle tantôt isolées, tantôt adossées à des associations (la Fédération des réseaux du parvis par exemple). Ils se retrouvent aussi dans des sessions organisées par des centres d'accueil comme il en existe beaucoup en France. Leur pensée est nourrie par des gens comme Marcel Légaut, mort en 1990, Bernard Feillet, Maurice Bellet ou le jésuite Joseph Moingt, par 
des philosophes comme Ricœur ou Lévinas. Ils restent attentifs à tout ce qui peut concourir à un renouveau de l'institution (ils se réjouissent par exemple des efforts déployés par le pape François) mais savent qu'elle restera toujours une superstructure et un lieu de pouvoir, dont ils ne contestent pas radicalement l'utilité, sans vouloir toutefois s'y associer. Ils cherchent avec d'autres, quelles que soient leurs orientations religieuses, morales ou spirituelles, des moyens à leur mesure pour répondre aux grands problèmes de l'heure : rénover la démocratie, répondre aux problèmes du chômage, de l'immigration de masse et du déclin de l'idée européenne. L'Évangile est toujours pour eux un texte vivifiant dans ses aspirations profondes, de sorte qu'on peut les désigner comme des croyants adogmatiques qui cherchent à pratiquer un humanisme évangélique. C'est là que s'ancre leur foi, dans la fidélité aux grandes aspirations des générations qui les ont précédés, des maîtres spirituels qui les ont formés, même s'ils n'ont plus, et de loin, les mêmes croyances. On a donc affaire à un christianisme partiellement ou totalement hors Église, dont la visibilité est modeste, mais que je crois vivant et fécond.

Où se situerait Jean par rapport à une telle évolution ? Comment aurait-il lui-même évolué ? Sans pouvoir évidemment répondre à une telle question, il me semble que lui, le solitaire mais aussi le bâtisseur en quête d'absolu, ne serait pas forcément mal à l'aise dans cette démarche, persévérante mais hors balisage, de recherche de sens et d'action. Elle n'est pas loin, au fond, de cette quête qui l'a conduit au baptême en 1959 : comme l'écrit Henry Bauchau («La femme de Samarie »), « il n’y a rien à espérer que l'espérance ». 


\section{Le livre n'est pas un produit comme les autres}

par Christian Thorel

N'est-ce pas sous cette affirmation que se passèrent les années de Jean Gattégno à la Direction du livre et au CNL ? Une question aujourd'hui : les années qui suivirent, particulièrement les années 2000, consacrent-elles l'union du même projet, de la même foi et des mêmes efforts de la part de ceux, éditeurs et libraires, qui s'abritent derrière cette doctrine?

Commençons par un rappel : sur le livre et sur son économie, il existait un constat, qui appelait une interprétation, il en découla un projet. Il fallut d'abord écrire une loi. Cette loi était la fondation d'un système plus complexe, que l'on disait « de régulation », elle était le premier acte d'une politique du livre. La promulguer, la mettre en place, était un risque. Parce que l'air du temps était celui des nouveaux modes de consommation, et particulièrement celui, idéologique et idéalisé, de la Fnac des origines. On sait d'ailleurs combien le terme "régulation » devint vite un gros mot dans le lexique des termes du néolibéralisme car ils s'introduisirent et s'imposèrent furtivement dans ce même temps.

La question de la loi à l'abri de laquelle se construisit le monde des livres que nous connaissons se prolonge désormais de celle de sa pérennité, plus exactement de la pérennité non de son objet, mais celle de son projet. Il me semble en effet que nous avons perdu la mémoire, que nous sommes installés dans l'évidence de la nécessité du prix unique, du caractère essentiel de la loi. Mais son essence, sa reconnaissance ? Comment aujourd'hui sont-elles lues, comprises, commentées, comment sont-elles interprétées? 
Énonçons quelques faits, rappelons que, tout comme l'esprit et l'organisation du droit d'auteur, le mode de fixation du prix des livres aura été de tout temps l'objet de débats, et souvent de litiges. Comment ignorer par exemple la confrontation de Jean Zay et de Bernard Grasset sur la propriété intellectuelle? Exemple de lutte de conceptions entre les plus hautes exigences du service public, le souci du bien commun, et celles des impératifs économiques, avérés et exagérés. Autre casse-tête, comment fixer le prix de vente au public d'un livre, produit manufacturé à haute valeur d'usage et à haute valeur symbolique, produit paradoxal d'un artisanat puis d'une industrie, transformant en nombre le fruit de la singularité d'une œuvre de l'esprit?

À la fin des années 1970, le travail associant Jérôme Lindon à François Mitterrand, à Jack Lang et à quelques instructeurs du socialisme qui advenait a été maintes fois commenté. Celui de Jean Gattégno l'a sans doute moins été. Il l'orchestra pourtant avec d'autres mesures pour la mise en œuvre d'une "politique du livre », et dans des directions complémentaires : soutien à la création, lecture publique, interprofession. Si l'un des objets de notre réunion est de témoigner de son action, c'est plutôt vers la loi sur le prix unique que je le ferai : sans doute faut-il que je répète que le principe, la vertu, associés à l'esprit de cette loi, étaient de neutraliser la concurrence par les prix pour mieux répondre à l'exigence qu'imposait le précepte : «Le livre n'est pas un produit comme les autres. » Ce postulat ne suffisait pas à emporter l'adhésion, pas plus que la loi qui en serait tirée, il fallait en effet la faire adopter, il fallait aussi la voter, il allait en sus falloir l'expliquer, il allait falloir la défendre. Non, ce ne devait en rien être une mesure exceptionnelle ou circonstancielle, mais le socle dense et inaliénable d'une économie politique du secteur. Nous allions entrer dans un temps de contestation et dans un temps de résistance. Si quelques hommes la conçurent, si quelques autres, plus nombreux, la promurent, d'autres plus 
nombreux encore la défendirent, parce qu'elle n'avait pas que des amis, pas que des partisans. On sait, mais on peut rappeler les noms des opposants les plus résolus, André Essel avec une conviction troublante, Michel-Édouard Leclerc avec l'arrogance de sa jeunesse, mais encore tous les anonymes, et autres velléitaires, tous les bonnets rouges, il y en avait déjà. Pour imposer le programme commun du livre, il fallait convaincre. Il fallait donc croire.

1981. Dans ce premier mois d'août du premier gouvernement Mauroy, Jérôme Lindon passa par Toulouse, c'était peu fréquent. Nous avions soutenu son action au sein de l'Association pour le prix unique (APU). Il ne nous prévint pas, et visita Ombres blanches une fin d'après-midi, inopinément. Un dîner improvisé fut l'occasion d'une leçon de politique générale du livre et de la culture. Nous aurions pu fêter le vote tout récent de la loi Lang, mais il préféra manifester son inquiétude envers la détermination des élus pour la défense du nouveau dispositif, tant ces mêmes élus faisaient l'objet d'une pression incessante depuis la direction de la Fnac. Il nous enjoignit de soutenir énergiquement la politique du ministère de la Culture, celle de la future Direction du livre, en déployant notre confiance en l'avenir par des actions commerciales, par des actions d'investissement. Il fallait en quelque sorte assumer une dette envers ceux, élus et fonctionnaires, envers le service public, qui misaient sur les générations futures et leur action dans le secteur du livre. Ce moment fut pour Ombres blanches la première de ses extensions et pour ses libraires comme une entrée véritable dans la vie professionnelle, la découverte de la responsabilité; ce fut aussi le début d'un compagnonnage et d'une alliance avec les Éditions de Minuit. De Toulouse à Paris et au-delà, nous n'étions pas les seuls à être impressionnés par la conviction de Jérôme Lindon et, même balbutiante, il se fit une prise de conscience générationnelle pouvant répondre aux exigences de l'éditeur et à 
celles du temps, et pouvant conforter la Direction du livre, Jean Gattégno et son équipe. Il est vrai que nous sommes alors dans un renouveau du commerce. Les grandes surfaces spécialisées (GSS) viennent compléter l'offre des hypermarchés. Et si, après les Trente Glorieuses, le livre restait la production de tête dans l'ordre du « commerce culturel » naissant, la crise qui succédait au choc pétrolier affectait les budgets des familles.

Et justement la crise pouvait légitimer tout ce qui favorisait la «baisse des prix » à la consommation, alors que l'engagement idéologique (et démagogique) de la Fnac, la « culture pour tous », avaient un grand pouvoir de séduction pour les élus locaux, obsédés par l'équipement commercial de leur ville, et la modernisation des villes. Il faut dire qu'en 1982, le modèle de la Fnac avait créé un sacré appel d'air, et un coup de blues aux librairies des années du gaullisme. Les jeunes libraires étaient peu nombreux, ils avaient les cheveux longs, un goût trop appuyé pour un certain désordre, les idées pas toujours bien rangées, mais aussi pas mal d'énergie. Avec ces bagages, il leur faudrait donc donner des preuves. «L'œil de la lettre » fut un bricolage associatif que nous montâmes d'abord à six, sous les directives de Jérôme Lindon et d'Henri Causse, et qui apporta dès 1984 un regard neuf sur le métier. Je persiste à croire que ce groupement, qui crût très vite, fut un événement politique, et qu'en dépit d'une importance relative sur le plan économique, il sut jouer des privilèges d'une avant-garde. « L'œil de la lettre » fut craint et aimé, et toujours sans rapport objectif avec sa taille et ses moyens. Il fut un lien avec l'édition et avec la Direction du livre, dans un environnement défaillant pour la représentation de la profession. La partie n'était pas que politique, la défense, la «protection » de l'économie du livre bénéficiaient d'un projet, attaché à celui de la singularité des livres, produits pas comme les autres. Si des groupements ont suivi la fin de « L'œil de la lettre », aucun n’a repris son activité de fer de lance pour la promotion de la loi 
Lang, la défense de la profession se faisant depuis près de vingt ans maintenant à partir du Syndicat de la librairie. Il est à noter tout de même que cette modification des représentations, sensible pour certains d'entre nous, n'a altéré en rien la détermination profonde des élus envers cette loi et la politique du livre, puisqu'ils les ont toujours renforcées. Le rapport Gaymard est de ce point de vue une approbation sans réserves de l'action de ceux, de celui, que nous saluons aujourd'hui.

Le public des lecteurs qui constituaient notre clientèle n'était pas toujours au fait des événements qui émaillaient la vie professionnelle, et «L'œil de la lettre » fut avant tout le lieu d'une expression collective de jeunes libraires qui manifestaient leur goût pour la littérature universelle en éditant de (presque) luxueux et exhaustifs catalogues de littératures dites encore " étrangères ». Ne retrouvait-on pas encore l'action de Jean Gattégno, tout autant que l'esprit dans lequel il conduisit sa mission? La traduction fut en effet une obsession bien connue du professeur de lettres anglaises. Autant la découverte des textes « du monde entier " que la reconnaissance de leurs traducteurs furent au programme du CNL qu'il présida. Imaginés par Jean-Paul Archie à Ombres blanches dès 1975, puis conçus par lui jusqu'à la dissolution du groupement, nos catalogues devinrent à leur façon la manifestation de notre responsabilité collective et d'un souci du don, d'un goût du bien commun, où se liaient nos commerces, les auteurs et les éditeurs des livres dont nos lecteurs attendaient que nous leur en livrions la connaissance. Par cet engagement culturel et éducatif, par cette production intellectuelle régulière, il nous semblait que nous écrivions les caractères les plus exemplaires de notre médiation. Probablement, et en usant d'un paradoxe, nous donnions ainsi à notre profession quelques lettres de noblesse tout autant que nous affirmions notre reconnaissance envers la République, à laquelle nous devions la possibilité de notre existence. 
Durant cette période de 1984 à 1990, j'ai eu la chance de passer des moments avec Jean Gattégno, moments autant privés que professionnels et dus à l'attention amicale de Patrice Béghain, DRAC à Toulouse entre 1986 et 1990. La figure de l'intellectuel, celle de l'écrivain, se superposent avec celle de l'homme du service public, exigeant, direct, naturellement autoritaire.

Mon dernier souvenir de Jean Gattégno est celui de l'inauguration de l'extension très importante de notre librairie en octobre 1989, et qui fut le moment d'un quasi-triplement de notre surface de vente. Ce passage, qui était un pari plus grand encore que celui de 1981, fut décisif et il nous fit franchir plusieurs étapes sur les plans professionnel et économique. Le 22 octobre de 1989, Jean Gattégno allait quitter la Direction du livre, mais n'avait pas hésité à venir présider l'ouverture de ce nouvel Ombres blanches, dans l'amitié. Il était accompagné d'une délégation tout aussi amicale d'éditeurs, lesquels venaient saluer un produit de la nouvelle structure de soutien à la librairie, qu'ils venaient de fonder, l'Association pour le développement de la librairie de création (Adelc). Le pari de la troisième version d'Ombres blanches ne pouvait en effet se réaliser qu'avec un accompagnement et une volonté, et nous avons eu la chance de pouvoir accéder à l'Adelc récemment créée. En 1988, Jean Gattégno n'hésita pas à se joindre au projet de la poignée d'éditeurs, édificateurs de ce mouvement. Tous ceux-là avaient-ils conscience de l'importance que prendrait la structure de soutien qu'ils mettaient en œuvre?

Depuis 1988, et en dépit des regards parfois soupçonneux, l'Adelc a donné sa chance à bien d'autres librairies, en préservant leur caractère d'indépendance. Savent-elles toutes l'origine de ce qui les soutient dans leur projet ? L'Adelc s'est inscrite naturellement dans la conduite de la politique du livre à la fin des années 1980 et, aussi redoutée et jalousée soit-elle, son action est plus validée 
encore que jamais. Parce que cette structure n'est pas une banque privée, encore moins un usurier, il serait bon que son action ne se solde pas dans une simple reconnaissance de dette, mais tout autant dans la dette d'une reconnaissance.

Si dans cette circonstance particulière à laquelle nous convie Jean Gattégno je fais ici appel à la connaissance de l'histoire par mes confrères, à la mémoire dont ils font défaut parfois, et à leurs préjugés, c'est que le sens d'une éthique se fait plus évident que jamais. Nous rappeler à notre sens de la responsabilité de libraires rend plus aisé d'exiger celui des éditeurs dont nous dépendons. Si le mot «indépendance » avait un sens, et je lui préfère celui d'" autonomie », ce serait pour qu'il nous aide à gagner de la reconnaissance dans l'environnement des livres et des lecteurs. Il me semble que le désir d'une responsabilité collective fut à l'œuvre dans l'action de Jean Gattégno, comme dans celle de ses successeurs. Surgit-elle en tous lieux de cet univers des livres qui est un mélange complexe, hétérogène, d'industrie, de finances, d'artisanat, de création?

Il y a tant de choses dites et écrites sur nos métiers, et tant de choses passionnantes provenant de l'histoire des livres. Avec Paul Angoulvent, fondateur des Presses universitaires de France (PUF) au milieu du siècle dernier, j'ai des désaccords concernant certaines de ses orientations politiques, mais je citerai pour finir un extrait de sa conclusion dans l'opuscule, consacré à ce même environnement, on dirait aujourd'hui "ce même écosystème ». Je le cite car j'y retrouve le goût pour la chose publique et pour l'engagement de Jean Gattégno, et ce mot provient d'un éditeur. C'est en 1960, et il écrit :

« Est-il un instant croyable que, sitôt le branle donné au développement en profondeur de l'instruction publique, les industries et commerces du Livre pourront se borner à faire passivement de bonnes affaires ? Il n'en est pas question. Transformés de gré 
ou de force en gardiens du feu du savoir, Éditeurs et Libraires, comme tous les gardiens du feu, en tout temps et en tout lieu, seront chargés de lourdes responsabilités. De plus en plus, on attendra d'eux, non de produire de beaux bilans, mais d'accomplir, moyennant une juste rémunération, le service que le peuple attend de leur dévouement au bien public.» 
Cet ouvrage a été réalisé avec Métopes, méthodes et outils pour l'édition structurée XML-TEI développés par le pôle Document numérique de la Maison de la recherche en sciences humaines de l'université de Caen.

Secrétariat d'édition :

Silvia Ceccani

Mise en page :

Nord Compo

Conception graphique :

atelier Perluette, 69001 Lyon

< http://www.perluette-atelier.com >

Le catalogue des presses

< http://www.enssib.fr/presses >

commander en ligne sur le comptoir des presses d'universités :

$<$ http://www.lcdpu.fr/editeurs/enssib/ >

ENSSib - UNIVERSITÉ DE LYON

PRESSES DE L'enssib

École nationale supérieure des sciences de l'information et des bibliothèques 17-21 boulevard du 11 novembre 1918 69623 Villeurbanne Cedex Tél. 0472444343 - Fax 0472444344

Contact : presses@enssib.fr

Première mise en ligne : septembre 2016.

Dépôt légal : $2^{\mathrm{e}}$ semestre 2016 